\title{
Critical Role for Isoprenoids in Apicoplast Biogenesis by Malaria Parasites
}

\section{Megan Okada', Krithika Rajaram², Russell P. Swift' ${ }^{2}$, Amanda Mixon', John Alan Maschek $^{3}$, Sean T. Prigge ${ }^{2}$, Paul A. Sigala ${ }^{*}$}

${ }^{1}$ Department of Biochemistry, University of Utah School of Medicine, Salt Lake City, Utah, United States; ${ }^{2}$ Department of Molecular Microbiology and Immunology, Johns Hopkins School of Public Health, Baltimore, Maryland, United States; ${ }^{3}$ Metabolomics Core, University of Utah Health Sciences Center, Salt Lake City, Utah, United States

*For correspondence: p.sigala@biochem.utah.edu 


\section{ABSTRACT}

49 Isopentenyl pyrophosphate (IPP) is an essential metabolic output of the apicoplast organelle in

50 Plasmodium falciparum malaria parasites and is required for prenylation-dependent vesicular

51 trafficking and other cellular processes. We have elucidated a critical and previously

52 uncharacterized role for IPP in apicoplast biogenesis. Inhibiting IPP synthesis blocks apicoplast

53 elongation and inheritance by daughter merozoites, and apicoplast biogenesis is rescued by

54 exogenous IPP and polyprenols. Knockout of the only known isoprenoid-dependent apicoplast

55 pathway, tRNA prenylation by MiaA, has no effect on blood-stage parasites and thus cannot

56 explain apicoplast reliance on IPP. However, we have localized an annotated polyprenyl synthase

57 (PPS) to the apicoplast lumen. PPS knockdown is lethal to parasites, rescued by IPP, and blocks

58 apicoplast biogenesis, thus explaining apicoplast dependence on isoprenoid synthesis. We

59 hypothesize that PPS synthesizes long-chain polyprenols critical for apicoplast membrane fluidity

60 and biogenesis. This work critically expands the paradigm for isoprenoid utilization in malaria

61 parasites and identifies a novel essential branch of apicoplast metabolism suitable for therapeutic

62 targeting.

63

64

65

66

67

68

69 
71 INTRODUCTION: Plasmodium falciparum malaria parasites are single-celled eukaryotes that

72 harbor a non-photosynthetic plastid organelle called the apicoplast which houses core metabolic

73 pathways and is essential for parasite viability. ${ }^{l}$ Because human cells lack this organelle and many

74 of its constituent enzymes, the apicoplast has been viewed as a potentially rich source of new

75 parasite-specific drug targets. However, cashing in on this potential has proved challenging, since

76 many apicoplast pathways, including heme $\mathrm{e}^{2,3}$ and fatty acid synthesis ${ }^{4,5}$, are dispensable during

77 parasite infection of erythrocytes when all malaria symptoms arise. Multiple antibiotics, including

78 doxycycline and clindamycin, block apicoplast biogenesis and inheritance and kill parasites, but

79 their slow activity over several lifecycles has been a fundamental limitation to broad clinical

80 application. ${ }^{6}$

81 A key, essential function of the apicoplast is biosynthesis and export of the isomeric

82 isoprenoid precursors, isopentenyl pyrophosphate (IPP) and dimethylallyl pyrophosphate

83 (DMAPP), via the non-mevalonate/methylerythritol phosphate (MEP) pathway. IPP and DMAPP,

84 which can be interconverted by an IPP isomerase, are critical for diverse cellular processes that

85 include prenylation of proteins involved in vesicular trafficking, dolichol-mediated protein

86 glycosylation, and biosynthesis of mitochondrial ubiquinone and heme A. ${ }^{7-10}$ Indeed, exogenous

87 IPP is able to rescue parasites from lethal apicoplast dysfunction or disruption, highlighting the

88 essential requirement for this isoprenoid precursor outside the apicoplast in blood-stage parasites. ${ }^{11}$

89 Consistent with these critical cellular roles for IPP, the MEP pathway inhibitor fosmidomycin

90 (FOS) kills parasites in the first lifecycle of treatment. $8,11,12$ This first-cycle FOS activity contrasts

91 with the delayed, second-cycle death observed for Plasmodium parasites treated with antibiotics

92 such as doxycycline and clindamycin that are thought to block translation of the 35-kb apicoplast

93 genome and the predominantly organelle-maintenance pathways it encodes. These contrasting 
94 kinetics have led to a prevailing view in the literature that essential apicoplast functions can be

95 segregated into two general categories: (1) anabolic pathways that produce metabolites required

96 outside the apicoplast and whose inhibition causes first-cycle parasite death or (2) housekeeping

97 pathways that are only required for organelle maintenance and whose inhibition causes delayed,

98 second-cycle defects. ${ }^{13-15}$ Although this simple paradigm has been useful for conceptualizing

99 general apicoplast functions, exceptions to this model have been reported ${ }^{12,16-18}$ and thus its

100 general validity remains uncertain.

101 Since exogenous IPP rescues parasites from lethal apicoplast disruption, ${ }^{11}$ isoprenoid

102 biosynthesis has been thought to only serve essential roles outside this organelle. ${ }^{7}, 12,15,16,19-21$

103 Indeed, P. falciparum expresses an essential cytoplasmic polyprenyl synthase (PF3D7_1128400)

104 whose dual farnesyl/geranylgeranyl pyrophosphate synthase (FPPS/GGPPS) activity is critical for

105 condensing isoprenoid precursors into longer polyprenyl-PP groups required for diverse cellular

106 processes such as protein prenylation and dolichol synthesis. ${ }^{19,}$ 22, 23 In addition, known

107 prenyltransferases, which attach prenyl groups such as FPP and GGPP to client proteins, are also

108 cytoplasmic. $^{20}$

109 In contrast to this prevailing paradigm, we have unraveled a novel essential arm of

110 isoprenoid metabolism and utilization within the apicoplast and provide direct evidence that IPP

111 and its condensation into downstream linear isoprenoids are required for apicoplast branching and

112 inheritance by daughter merozoites. Genetic knockout of MiaA-dependent tRNA prenylation, the

113 only previously predicted isoprenoid-dependent pathway in the apicoplast, ${ }^{l}$ has no effect on blood-

114 stage parasites, and thus MiaA cannot account for apicoplast dependence on IPP. However, we

115 have localized a previously annotated polyprenyl synthase (PPS, PF3D7_0202700) ${ }^{24}$ to the

116 apicoplast lumen and show that its conditional knockdown is lethal to parasites, can be rescued by 
117 IPP and long- but not short-chain polyprenols, and blocks apicoplast inheritance. We posit that

118 this apicoplast PPS functions downstream of IPP synthesis to produce longer-chain isoprenoids

119 essential for apicoplast membrane fluidity during organelle biogenesis. This discovery critically

120 expands the paradigm for isoprenoid utilization in $P$. falciparum, identifies a potential new

121 apicoplast drug target, and uncovers an organelle maintenance pathway whose inhibition causes

122 first-cycle defects in apicoplast inheritance in contrast to delayed death-inducing antibiotics.

124 RESULTS

125 Apicoplast elongation and branching require isoprenoid precursor synthesis. The $P$.

126 falciparum literature has focused almost exclusively on the essential roles of isoprenoid

127 metabolism outside the apicoplast. ${ }^{7}, 11,14,15,19,20$ Nevertheless, several prior studies reported that

128 MEP-pathway inhibitors such as FOS and MMV008138 blocked apicoplast elongation in lethally

129 treated parasites, suggesting a possible role for IPP in apicoplast biogenesis. ${ }^{25-27}$ These prior

130 studies, however, could not rule out that defects in apicoplast development caused by MEP-

131 pathway inhibitors were due to non-specific effects from the pleiotropic cellular dysfunctions

132 inherent to parasite death. ${ }^{19}$ We revisited FOS inhibition of apicoplast biogenesis to further test

133 and distinguish specific versus non-specific effects on organelle development.

134 We first tested the effect of $10 \mu \mathrm{M}$ FOS $\left(10 \mathrm{x} \mathrm{EC}_{50}\right)$ on apicoplast elongation in

135 synchronized cultures of two different parasite strains: D10 parasites expressing the apicoplast-

136 targeted acyl carrier protein (ACP) leader sequence fused to GFP $\left(\mathrm{ACP}_{\mathrm{L}-\mathrm{GFP}}\right)^{28}$ and a recently

137 published NF54 parasite line (PfMev) that expresses $\mathrm{ACP}_{\mathrm{L}-\mathrm{GFP}}$ as well as heterologous enzymes

138 that enable cytoplasmic synthesis of IPP from exogenous mevalonate precursor, independent of

139 the apicoplast MEP pathway. ${ }^{29}$ Consistent with prior reports, ${ }^{25-27,30}$ we observed that synchronized 
140 ring-stage parasites treated with FOS developed into multi-nuclear schizonts but failed to elongate

141 the apicoplast, which retained a focal, unbranched morphology in PfMev (Figure 1A) and D10

142 parasites (Figure 1- figure supplements 1-3). Although MEP pathway activity is detectable in ring-

143 stage parasites, ${ }^{31,32}$ identical inhibition of apicoplast elongation in schizonts was observed if FOS

144 was added to trophozoites 12 hours after synchronization (Figure 1, A and C, and Figure 1- figure

145 supplement 2), suggesting continued reliance on de novo synthesis. In contrast to FOS treatment,

146 parasites treated with lethal doses $\left(10-100 \mathrm{x} \mathrm{EC}_{50}\right)$ of drugs that target processes outside the

147 apicoplast, including DSM1 (mitochondrial dihydroorotate dehydrogenase inhibitor), ${ }^{33}$

148 atovaquone (ATV, mitochondrial cytochrome b inhibitor), ${ }^{34}$ blasticidin-S (Blast-S, cytoplasmic

149 translation inhibitor), ${ }^{35}$ or WR99210 (WR, cytoplasmic dihydrofolate reductase inhibitor), ${ }^{36}$

150 exhibited normal apicoplast biogenesis as they developed into schizonts, very similar to untreated

151 parasites (Figure 1, B and C, and Figure 1- figure supplement 2). These observations strongly

152 suggest that defects in apicoplast elongation observed with FOS treatment are due to specific

153 inhibition of MEP pathway activity rather than non-specific, secondary effects of parasite death.

155 exogenous IPP and do not show evidence of apicoplast loss, ${ }^{11,12,21,29}$ suggesting that IPP rescues

156 apicoplast biogenesis from FOS-induced defects. To directly test this conclusion, we

157 simultaneously treated synchronized rings with $10 \mu \mathrm{M}$ FOS and either $200 \mu \mathrm{M}$ IPP or $50 \mu \mathrm{M}$

158 mevalonate (for PfMev parasites) and observed normal apicoplast elongation and branching in

159 schizonts (Figure 1, A and C, and Figure 1- figure supplement 2), consistent with a prior report. ${ }^{26}$

160 These observations directly support the conclusion that apicoplast elongation requires isoprenoid

161 synthesis. 
To further test this conclusion via genetic disruption rather than pharmacological

163 inhibition, we utilized a previously reported line of PfMev parasites in which the apicoplast-

164 targeted deoxyxylulose-5-phosphate synthase (DXS), the first enzyme in the MEP isoprenoid

165 synthesis pathway, had been genetically deleted ( $\triangle \mathrm{DXS}) .{ }^{37}$ These parasites require exogenous

167 pathway. In the presence of $50 \mu \mathrm{M}$ mevalonate, $\Delta \mathrm{DXS}$ parasites displayed normal apicoplast

168 elongation and branching in schizonts. However, washing out mevalonate from ring-stage $\Delta \mathrm{DXS}$

169 parasites to ablate IPP synthesis resulted in multinuclear schizonts with focal, unbranched

170 apicoplast morphologies identical to those observed in the presence of FOS (Figure 1, D and E,

171 and Figure 1- figure supplement 4). These results strongly support the conclusion that apicoplast

172 elongation and branching require IPP synthesis.

A.
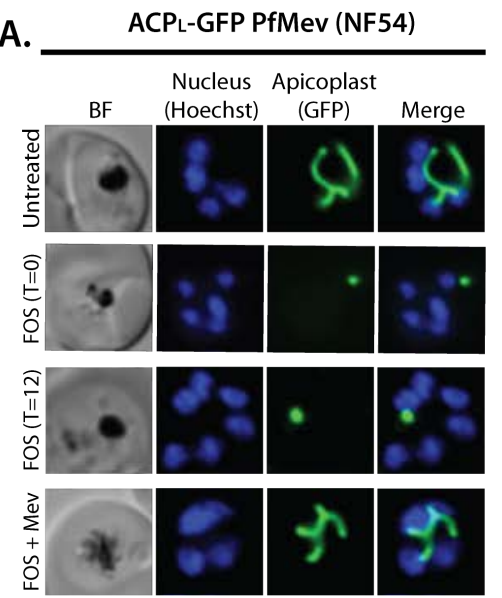

D.

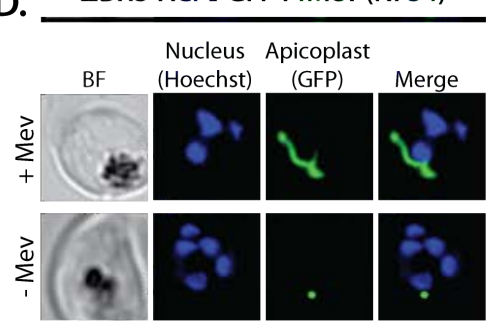

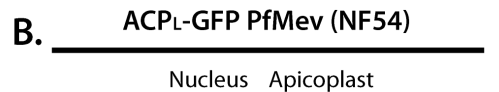
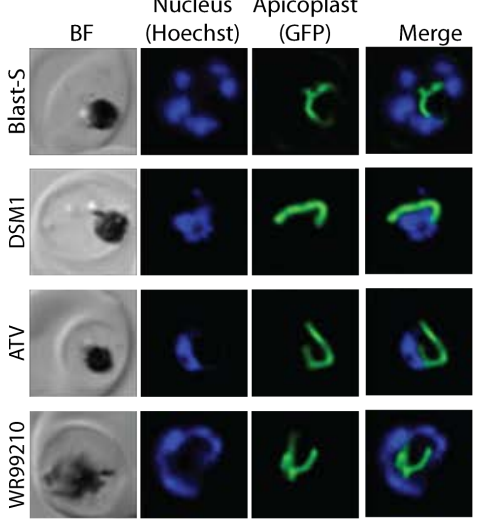

E. $\triangle$ DXS PfMev Apicoplast Status

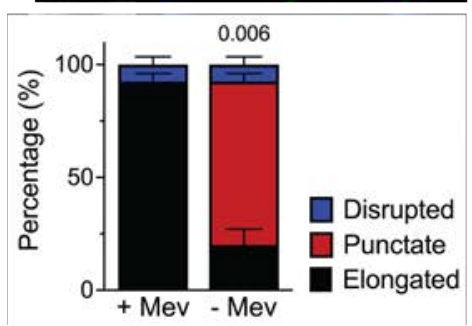

C. ACPL-GFP PfMev (NF54) Apicoplast Status

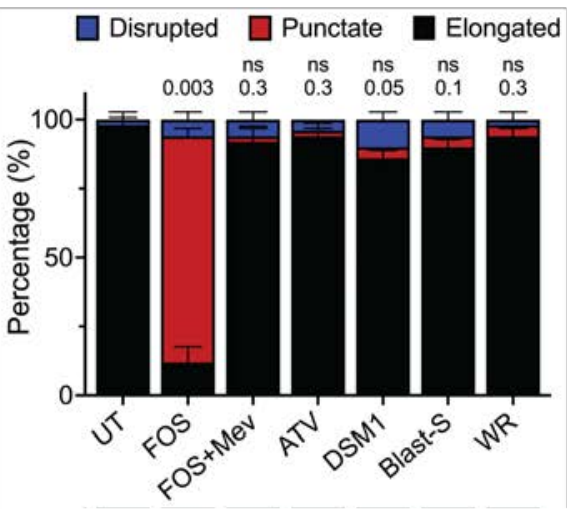

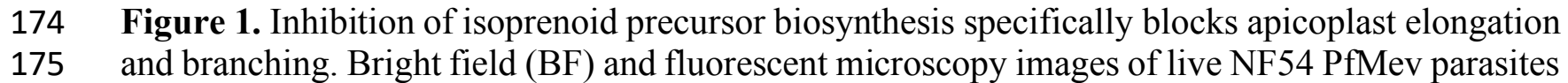
176 that were (A) untreated or treated with $10 \mu \mathrm{M}$ fosmidomycin (FOS) in the absence or presence of 
$50 \mu \mathrm{M}$ DL-mevalonate (Mev), or (B) treated with $6 \mu \mathrm{M}$ blasticidin-S (Blast-S), $2 \mu \mathrm{M}$ DSM1, 100 $\mathrm{nM}$ atovaquone (ATV), or $5 \mathrm{nM}$ WR99210. (C) Statistical analysis of apicoplast morphology for 50 total parasites imaged for each condition in panels A and B from two independent experiments. Apicoplast morphologies were scored as punctate (focal), elongated, or disrupted (dispersed); counted; and plotted by histogram as the fractional population with the indicated morphology. Error bars represent standard deviations from replicate experiments. Two-tailed unpaired t-test analysis was used to determine the significance of observed population differences compared to untreated (UT) parasites (P values given above each condition, ns $=$ not significant). (D) Live-cell imaging of $\triangle \mathrm{DXS}$ PfMev parasites in the presence or absence of $50 \mu \mathrm{M}$ Mev. (E) Statistical analysis of parasites imaged in panel $\mathrm{D}$ and performed as in panel $\mathrm{C}$. The $\mathrm{P}$ value is for comparison of apicoplast morphologies $\pm \mathrm{Mev}$ conditions. In all experiments, synchronized ring-stage parasites were incubated with the indicated treatments for 36 hours prior to live-cell imaging. Parasite nuclei were visualized using $1 \mu \mathrm{g} / \mathrm{ml}$ Hoechst 33342 . The parasite apicoplast was visualized using the $\mathrm{ACP}_{\mathrm{L}}$-GFP encoded by the PfMev line. Absolute parasite counts for all microscopy experiments are shown in Figure 1- Source Data 1.

Figure supplement 1. Additional epifluorescence images of PfMev NF54 parasites treated with FOS and other drugs.

Figure supplement 2. Epifluorescence images and analysis of D10 $\mathrm{ACP}_{\mathrm{L}}-\mathrm{GFP}$ parasites treated with FOS and other drugs.

Figure supplement 3. Additional epifluorescence images of D10 parasites treated with FOS and other drugs.

Figure supplement 4. Additional epifluorescence images of $\triangle \mathrm{DXS}$ PfMev parasites cultured $\pm \mathrm{Mev}$.

Source data 1. Absolute parasite counts for all microscopy experiments.

\section{Inhibition of isoprenoid synthesis prevents apicoplast inheritance by daughter parasites. To}

stringently test that IPP synthesis is required for apicoplast biogenesis, we next asked if FOS treatment prevented daughter parasites from inheriting the apicoplast, as predicted to occur if the apicoplast fails to elongate and divide in schizonts and as commonly observed for antibiotic inhibitors of apicoplast housekeeping pathways. ${ }^{12}, 38$ Simultaneous treatment of ring-stage parasites with both FOS and IPP rescued growth defects and resulted in normal apicoplast elongation and division (Figure 1A and Figure 1- figure supplement 1), as expected since IPP is the direct anabolic product of the MEP pathway specifically inhibited by FOS. Thus, concomitant treatment with IPP and FOS cannot distinguish whether MEP pathway inhibition prevents 
211 apicoplast inheritance by daughter parasites. To bypass this fundamental limitation, we devised

212 the following alternative strategy.

The apicoplast begins to elongate near the onset of schizogony before branching and then

214 dividing in late, segmenting schizonts. ${ }^{28,39}$ Despite manifesting defects in apicoplast elongation in

215 early schizogony, FOS-treated parasites continue to divide nuclear DNA and transition into mature

216 schizonts before stalling prior to segmentation into merozoites (Figure 1A). ${ }^{30}$ This observation

217 suggested that defects in apicoplast biogenesis were not the immediate cause of parasite death in

218 the present cell cycle and that such defects preceded a broader essential requirement for IPP outside

219 the apicoplast in mature schizonts. Recent works suggest this broader essentiality to be IPP-

220 dependent protein prenylation. ${ }^{15,30}$ We therefore reasoned that if IPP supplementation were

221 delayed until mid-schizogony, after the onset of apicoplast elongation defects but before broader

222 cellular death, it might be possible to rescue parasite viability without rescuing apicoplast

223 biogenesis and thereby produce viable parasite progeny that lacked the intact apicoplast.

$22550 \mu \mathrm{M}$ mevalonate added at $0,30,34$, or 38 hours after synchronization. (Figure 2A). Parasites

226 were allowed to expand for three subsequent cycles in $50 \mu \mathrm{M}$ mevalonate, with growth monitored

227 by flow cytometry. We observed a hierarchy of growth rescue by mevalonate, with full rescue

228 (relative to no FOS treatment) of parasites supplemented with mevalonate at 0 hours post-

229 synchronization and decreasing rescue for increasingly delayed supplementation at 30,34 , or 38

230 hours (Figure 2B), presumably due to fewer viable parasites surviving the initial cycle.

To assess and quantify apicoplast status in rescued parasites, we cloned out individual

232 parasites at 60 hours post-synchronization in the second growth cycle. Apicoplast status in the

233 resulting clones was determined by live parasite microscopy of organelle morphology, apicoplast 
234 genome PCR, and growth \pm mevalonate. Although FOS-treated parasites supplemented

235 simultaneously with mevalonate showed no evidence for apicoplast loss in clonal progeny, a

236 fraction of clonal parasites derived from delayed mevalonate rescue showed clear signs of

237 apicoplast loss, including a dispersed apicoplast $\mathrm{ACP}_{\mathrm{L}}$-GFP signal, loss of the apicoplast genome,

238 and growth dependence on exogenous mevalonate (Figure 2C and Figure 2- figure supplements

239 1-4). The fraction of clonal parasites with a disrupted apicoplast increased from 10\% in parasites

240 supplemented with mevalonate at 30 hours to over $80 \%$ in parasites supplemented at 38 hours

241 (Figure 2D). These results provide direct evidence that inhibiting IPP synthesis alone is sufficient

242 to block apicoplast biogenesis and prevent organelle inheritance by daughter parasites.

A.

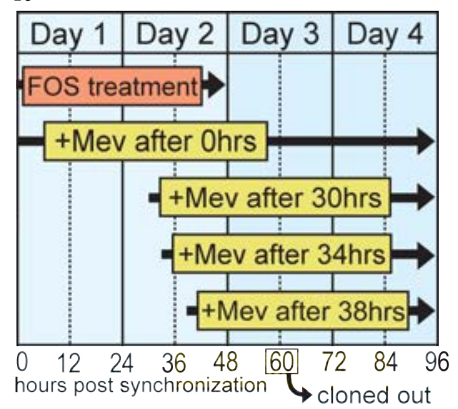

C.

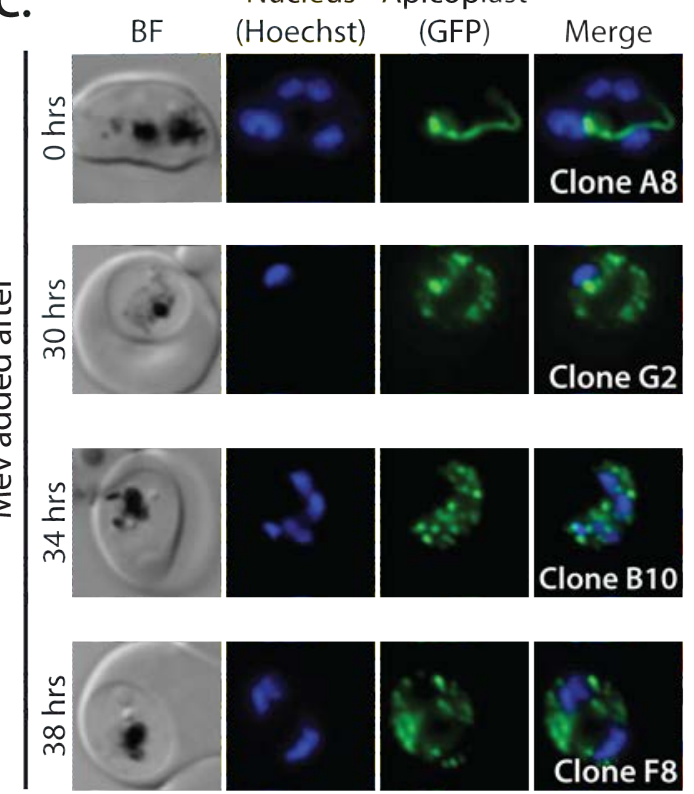

B.

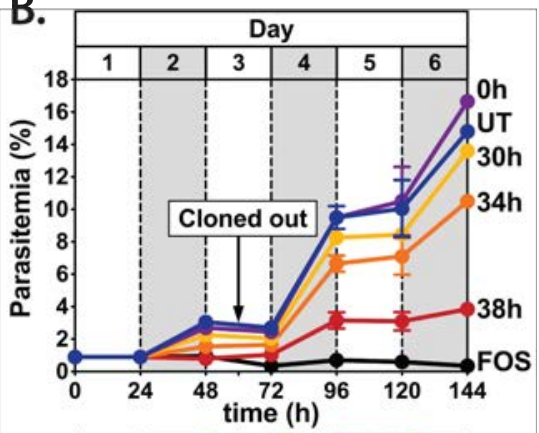

$\frac{\text { Genome PCR }}{\mathrm{Nu} \text { Api }}$
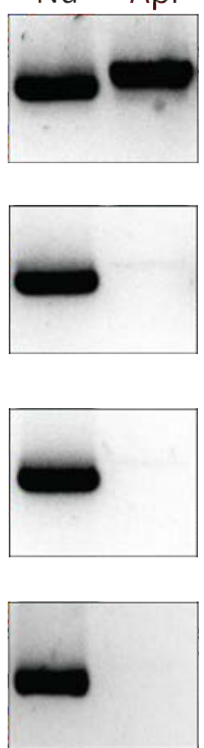

D. Intact $\square$ Disrupted

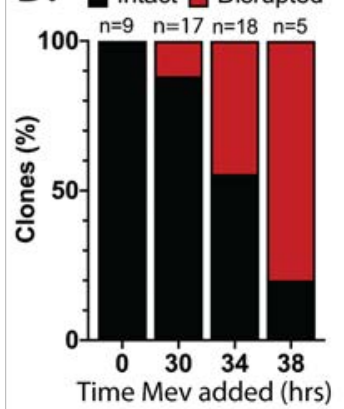

Growth Assay

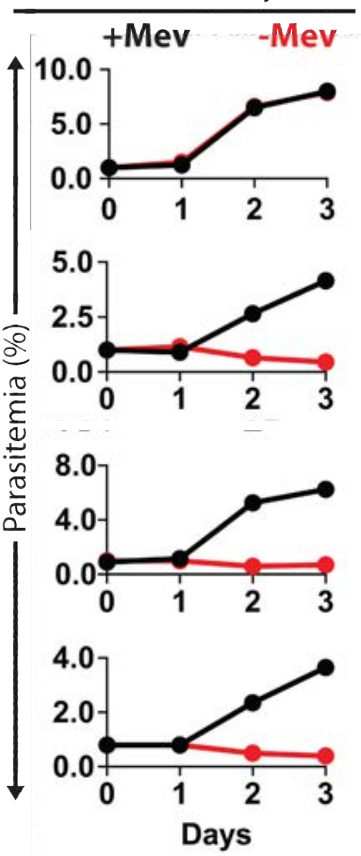


Figure 2. Inhibiting isoprenoid precursor biosynthesis prevents apicoplast inheritance by daughter parasites. (A) Schematic summary of delayed mevalonate rescue of fosmidomycin (FOS) treatment. PfMev parasites were synchronized with 5\% D-sorbitol and cultured in $10 \mu \mathrm{M}$ FOS (washed out after 48 hours in $2^{\text {nd }}$-cycle rings) without or with addition of $50 \mu \mathrm{M}$ DL-mevalonate (Mev) at $0,30,34$, or 38 hours after synchronization. Clonal parasites from all growth conditions were isolated at 60 hours post-synchronization by limiting dilution and growth in $50 \mu \mathrm{M} \mathrm{Mev}$. (B) Parasite growth was monitored for 6 days by flow cytometry using acridine orange staining (FOS $=$ treated only with FOS, UT $=$ untreated, $0 \mathrm{~h}-38 \mathrm{~h}$ time delay of Mev addition after synchronization and initiation of FOS treatment). (C) Bright-field (BF) and fluorescence microscopy images of live clonal parasites isolated after 60 hours of growth under the conditions described in panel A. Parasite nuclei were visualized using $1 \mu \mathrm{g} / \mathrm{ml}$ Hoechst 33342 . To the right of each clonal image panel is a gel image showing the result of PCR analysis to amplify a $(\mathrm{Nu})$ nuclear (PPS, PF3D7_0202700) and (Api) apicoplast (SufB, PF3D7_API04700) gene and a growth assay to monitor the ability of each isolated clone in the presence or absence of $50 \mu \mathrm{M}$ Mev. Data points are the average of two technical replicates from a single growth assay. Error bars (stdev) are smaller than the data points. (D) Graphical representation of the number of clones isolated under each growth condition and the clonal percentage with an intact or disrupted apicoplast (determined by microscope analysis of $\mathrm{ACP}_{\mathrm{L}}-\mathrm{GFP}$ signal and genomic PCR).

Figure supplement 1. Epifluorescence microscopy images of clonal parasites isolated after FOS treatment and rescue by mevalonate addition at 0 hours after synchronization.

Figure supplement 2. Epifluorescence microscopy images of clonal parasites isolated after FOS treatment and rescue by mevalonate addition at 30 hours after synchronization. Clone headers for parasites with a disrupted apicoplast are yellow.

Figure supplement 3. Epifluorescence microscopy images of clonal parasites isolated after FOS treatment and rescue by mevalonate addition at 34
parasites with a disrupted apicoplast are yellow.

Figure supplement 4. Epifluorescence microscopy images of clonal parasites isolated after FOS treatment and rescue by mevalonate addition at 38 hours after synchronization. Clone headers for parasites with a disrupted apicoplast are yellow.

The MiaA pathway for apicoplast tRNA prenylation is dispensable for blood-stage parasites.

Why do apicoplast elongation and branching require IPP synthesis? Currently, the only predicted 
genes encoding MiaA (PF3D7_1207600) and MiaB (PF3D7_0622200) are annotated in the $P$.

falciparum genome and MiaA protein has been detected by mass spectrometry in the apicoplast-

284 specific proteome, ${ }^{42}$ neither protein has been directly studied in parasites. Nevertheless, both

285 proteins are predicted to be non-essential for blood-stage Plasmodium based on genome-wide knockout (KO) studies in P. berghei ${ }^{43}$ and P. falciparum. ${ }^{44}$

To directly test whether MiaA function is essential for $P$. falciparum parasites and can account for apicoplast dependence on isoprenoid synthesis, we used CRISPR/Cas9 to target MiaA

289 for gene disruption by double-crossover homologous recombination (Figure 3- figure supplement

290 1). PfMev parasites were transfected and selected in the presence of $50 \mu \mathrm{M}$ mevalonate to ensure

291 that parasites would remain viable even if deletion of MiaA resulted in apicoplast disruption.

292 Parasites that had integrated the knock-out plasmid returned from transfection, and loss of the

293 MiaA gene was confirmed by genomic PCR (Figure 3- figure supplement 1). The $\Delta$ MiaA parasites

294 grew equally well in the presence or absence of mevalonate and grew indistinguishably from the

295 parental PfMev parasites (Figure 3A). The presence of an intact apicoplast was confirmed by

296 genomic PCR analysis and live parasite microscopy (Figure 3B and Figure 3- figure supplement

297 1). These results indicate that MiaA is dispensable for blood-stage parasites and that deletion of

298 this gene does not affect apicoplast biogenesis. Therefore, loss of function of MiaA, the only

299 predicted isoprenoid-dependent pathway in the apicoplast, cannot account for apicoplast

300 dependence on IPP synthesis, suggesting an alternative role for IPP in organelle elongation. 


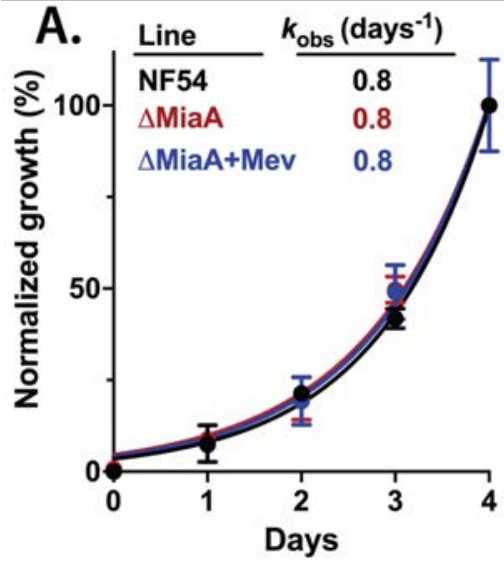
(SufB, PF3D7_API04700). images of apicoplast morphology.
B.

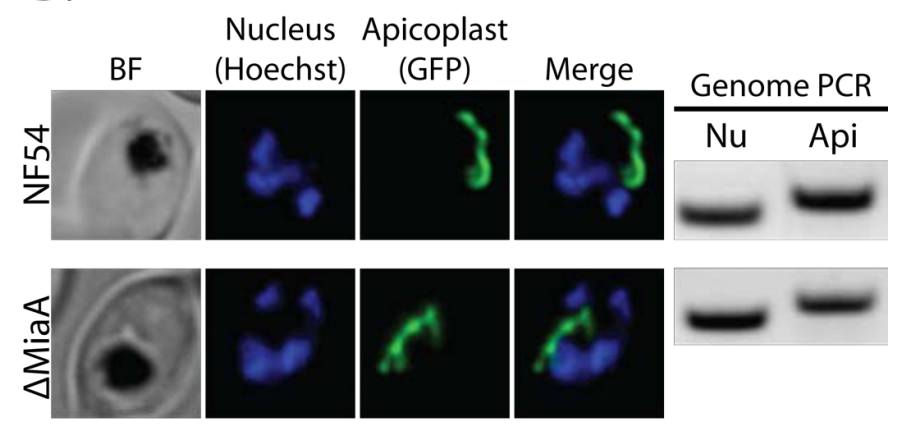

Figure 3. Genetic disruption of MiaA has no effect on parasite growth or apicoplast biogenesis. (A) Growth analysis indicates that parental PfMev NF54 parasites and $\Delta$ MiaA parasites cultured in the absence or presence of $50 \mu \mathrm{M}$ Mev grow indistinguishably with identical rate constants $\left(k_{\text {obs }}\right)$ for asynchronous culture expansion. Parasitemia values for each sample are the average \pm SD of three biological replicates and were normalized to the parasitemia on day 4 and fit with an exponential growth model. (B) Live-parasite imaging and genomic PCR analysis indicate normal apicoplast morphology and retention of the apicoplast genome in parental PfMev and $\Delta \mathrm{MiaA}$ parasites. $\mathrm{BF}=$ brightfield, $\mathrm{Nu}=$ nuclear gene $\left(\mathrm{LDH}, \mathrm{PF} 3 \mathrm{D} 7 \_1324900\right)$, and Api $=$ apicoplast gene

Figure supplement 1. PCR genotyping of PfMev $\triangle \mathrm{MiaA}$ parasites and additional epifluorescence

Apicoplast biogenesis requires polyprenyl isoprenoid synthesis. Except for MiaA-catalyzed

tRNA prenylation, all proposed roles for isoprenoids in Plasmodium parasites require head-to-tail

condensation of DMAPP (5 carbons) and one or more IPP subunits (5 carbons) to form longerchain isoprenoids, starting with formation of geranyl pyrophosphate (GPP, 10 carbons), farnesyl pyrophosphate (FPP, 15 carbons), and geranylgeranyl pyrophosphate (GGPP, 20 carbons). ${ }^{\text {7, }} 20$ Recent studies reported that $5 \mu \mathrm{M}$ geranylgeraniol (GGOH, the alcohol precursor of GGPP) can provide short-term $(\sim 1$ cycle) rescue of parasite death due to treatment with FOS or indolmycin, an apicoplast tryptophan tRNA synthetase inhibitor. ${ }^{15,30}$ Based on these reports, we hypothesized 
325 isoprenoids such that farnesol $(\mathrm{FOH}), \mathrm{GGOH}$, and/or longer-chain polyprenols might rescue the

326 apicoplast branching defects caused by $10 \mu \mathrm{M}$ FOS.

$328 \mathrm{FOH}$ or GGOH. Consistent with prior reports, $5 \mu \mathrm{M} \mathrm{GGOH}$ but not FOH partially rescued parasite

329 growth from inhibition by FOS and enabled culture expansion into a second growth cycle (Figure

330 4- figure supplement 1). Nevertheless, both GGOH and FOH rescued apicoplast elongation and

331 branching defects in schizonts when added simultaneously with FOS to synchronized rings (Figure

3324 and Figure 4- figure supplements 2 and 3). Rescue of apicoplast branching by FOH and GGOH

333 strongly suggests that apicoplast biogenesis depends on utilization of polyprenyl isoprenoids of

334 three or more isoprene units. We extended these rescue experiments to include $5 \mu \mathrm{M}$ decaprenol

335 (50 carbons) and also observed rescue of apicoplast branching from FOS-induced defects.

336 However, $5 \mu \mathrm{M} \beta$-carotene, which is a nonlinear carotenoid hydrocarbon derived from 8 prenyl

337 groups (40 carbons), did not rescue apicoplast biogenesis from inhibition by FOS (Figure 4 and

338 Figure 4- figure supplements 2 and 3). These results directly suggest that apicoplast biogenesis

339 specifically requires synthesis of linear polyprenols containing three or more prenyl groups.

341 polyprenyl-PPs requires the function of a polyprenyl synthase. This family of enzymes uses a

342 conserved dyad of DDXXD residues positioned near the protein surface of the active site binding

343 pocket to coordinate $\mathrm{Mg}^{2+}$ ions that bind the pyrophosphate headgroup of DMAPP, GPP, or FPP

344 and position its allylic head relative to the vinyl tail of the IPP subunit. ${ }^{45}$ Condensation of the two

345 substrates via electrophilic alkylation elongates the nascent isoprenoid chain into the protein

346 interior. Two amino acids just upstream of the first DDXXD motif determine the length of the

347 resulting prenyl chain by forming a hydrophobic "floor" that gates the depth of the protein interior. 
Indeed, dedicated FPPS enzymes feature an amino acid floor comprised of sequential Phe-Phe residues just upstream of the first DDXXD motif that sterically block synthesis of products longer than FPP. ${ }^{46,47}$ Sequence variations that replace just the more N-terminal Phe or both Phe-Phe groups with smaller residues (e.g., Ala or Ser) open up and extend the binding pocket and enable synthesis of GGPP or longer polyprenyl-PPs up to 14 isoprene units, respectively (Figure 5). ${ }^{48}$

A BLAST search of the $P$. falciparum genome with the sequence of the well-studied

354 chicken FPP synthase (Uniprot P08836) reveals two parasite orthologs (PF3D7_1128400 and PF3D7_0202700) that retain the DDXXD dyads and other conserved sequence features expected of a polyprenyl synthase (Figure 5A and Figure 5- figure supplement 1). The best studied of these synthases is the cytosolic enzyme, PF3D7_1128400, which shares 34\% sequence identity with avian FPPS and has been reported to catalyze formation of both FPP and GGPP. ${ }^{22,49,50}$ Consistent with its ability to synthesize GGPP as the terminal product, PF3D7_1128400 has sequential SerPhe residues just upstream of the first DDXXD motif (Figure 5A). ${ }^{49}, 50$ This cytoplasmic enzyme

361 is reported to be essential based on inhibitor ${ }^{19,23}$ and gene-disruption studies in P. berghei $i^{43}$ and P. falciparum $^{44}$ and is thought to synthesize the FPP and GGPP required for broad parasite

363 isoprenoid metabolism, including protein prenylation and synthesis of dolichols, ubiquinone, and

364 heme A. 7,20

We first considered the model that this cytoplasmic FPPS/GGPPS might have an essential role in producing GGPP required for apicoplast biogenesis. A recent study, however, identified a

367 specific inhibitor (MMV019313) of PF3D7_1128400 that is lethal to parasites but does not impact 368 apicoplast biogenesis. ${ }^{19}$ We independently confirmed that lethal treatment with MMV019313 did not affect apicoplast branching in the PfMev line (Figure 5- figure supplement 2). These 
synthase activity required for apicoplast biogenesis. Therefore, we turned our attention to the

373 sequence identity with avian FPPS.

A.
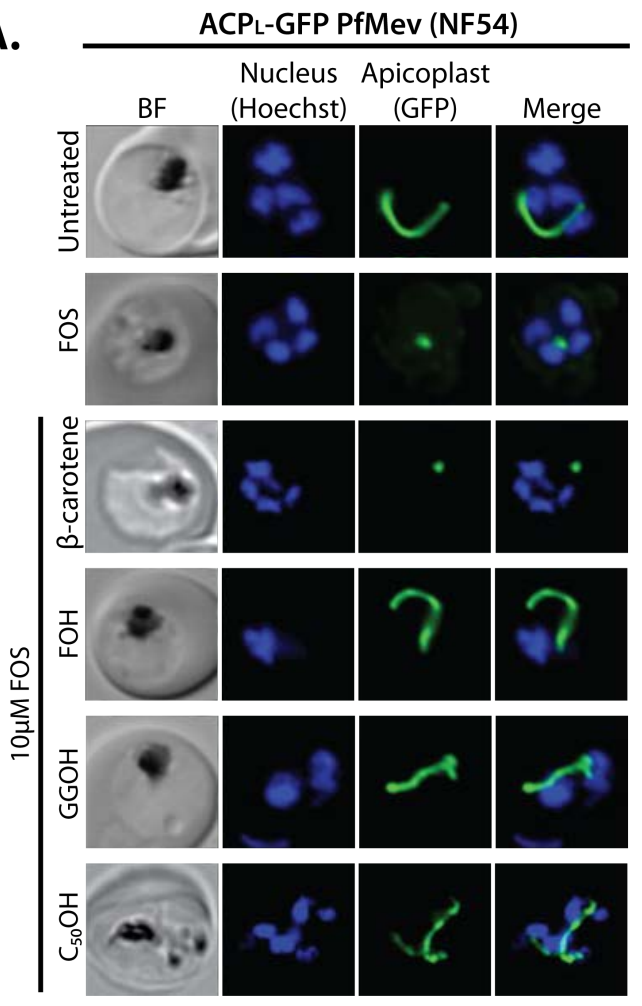

\section{B. ACPL-GFP PfMev (NF54) Apicoplast Status}

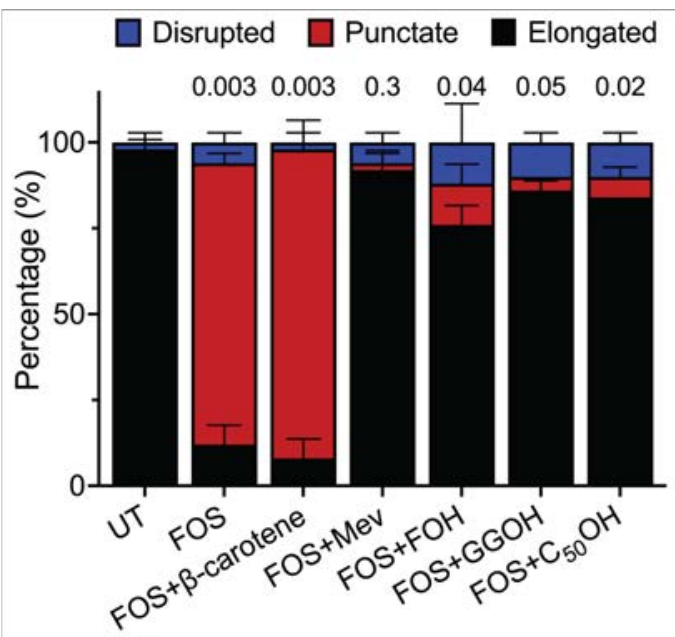

Figure 4. Apicoplast biogenesis requires linear polyprenyl isoprenoid synthesis. (A) $5 \mu \mathrm{M}$ farnesol (FOH), geranylgeraniol $(\mathrm{GGOH})$, or decaprenol $\left(\mathrm{C}_{50}-\mathrm{OH}\right)$, but not $\beta$-carotene rescues apicoplast biogenesis from inhibition by $10 \mu \mathrm{M}$ FOS in PfMev parasites. Synchronized ring-stage parasites were incubated with the indicated treatments for 36 hours and imaged by brightfield (BF) or fluorescence microscopy, with visualization of parasite nuclei by Hoechst staining and the apicoplast by $\mathrm{ACP}_{\mathrm{L}}$-GFP signal. (B) Statistical analysis of apicoplast morphology for 50 total parasites imaged for each condition in panel A from two independent experiments. Apicoplast morphologies were scored as punctate (focal), elongated, or disrupted (dispersed); counted; and plotted by histogram as the fractional population with the indicated morphology. Error bars represent standard deviations from replicate experiments. Two-tailed unpaired t-test analysis was used to determine the significance of observed population differences compared to untreated (UT) parasites ( $\mathrm{P}$ values given above each condition, $\mathrm{ns}=$ not significant).

Figure supplement 1. $5 \mu \mathrm{M}$ GGOH but not FOH partially rescues parasite growth from inhibition by $10 \mu \mathrm{M}$ FOS in continuous-growth assays with PfMev parasites.

Figure supplement 2. Additional epifluorescence microscopy images of PfMev parasites treated with $\mathrm{FOS}$ and $\mathrm{FOH}, \mathrm{GGOH}, \mathrm{C}_{50}-\mathrm{OH}$, or $\beta$-carotene. 
Figure supplement 3. Epifluorescence microscopy images of D10 ACP $\mathrm{A}_{\mathrm{L}} \mathrm{GFP}$ parasites treated with FOS and $\mathrm{FOH}, \mathrm{GGOH}, \mathrm{C}_{50}-\mathrm{OH}$, or $\beta$-carotene.

Localization of an annotated polyprenyl synthase to the apicoplast. Like the cytoplasmic

FPP/GGPP synthase, PF3D7_0202700 retains the DDXXD sequence dyad expected for a polyprenyl pyrophosphate synthase. In addition, the amino acid floor of PF3D7_0202700 features a sterically smaller Gly-Ser dyad upstream of the first DDXXD (Figure 5A and figure 5- figure supplement 1) that suggests an ability to synthesize longer-chain isoprenoids greater than four 401 isoprene units. Consistent with these features, sequence similarity searches via NCBI BLAST ${ }^{51}$ 402 and MPI HHpred ${ }^{52}$ identify polyprenyl synthase homologs from bacteria, algae, and plants that 403 share $\sim 30 \%$ sequence identity with PF3D7_0202700 and have annotated functions in synthesizing 404 polyprenyl isoprenoids of 4 - 10 units (Figure 5- figure supplement 3). Using E. coli octaprenyl 405 pyrophosphate synthase (PDB $3 \mathrm{WJK}, 28 \%$ identity) as template, we generated a homology model 406 of PF3D7_0202700 to visualize the possible structure of its active site (Figure 5B).

410 protein appears to have lacked one of the DDXXD motifs. Because of this difference from the

411 native protein and the impurity of the parasite-derived protein, it remains possible that the native,

412 pure protein has a distinct product spectrum than previously reported. Nevertheless, this in vitro 413 activity and the general sequence features of PF3D7_0202700 support its function as a long-chain 414 polyprenyl synthase (PPS). 
417 cellular compartment. ${ }^{53}$ Analysis of the protein sequence with PlasmoAP ${ }^{54}$ suggested the presence

418 of a subcellular-targeting leader sequence, with strong prediction of an apicoplast-targeting transit

419 peptide but uncertainty in the presence of a signal peptide. To localize PPS within parasites, we

420 engineered Dd2 P. falciparum lines to episomally express full-length PPS fused to either C-

421 terminal GFP or RFP. In live parasites, focal PPS-GFP fluorescence was detected in a tubular

422 compartment proximal to but distinct from the mitochondrion, as expected for apicoplast

423 localization. Additional immunofluorescence analysis of the PPS-GFP line revealed strong co-

424 localization between PPS-GFP and the apicoplast acyl carrier protein (ACP) (Figure 5C and Figure

425 5- figure supplement 4).

To further confirm apicoplast targeting of PPS, we stably disrupted the apicoplast in the

427 PPS-GFP Dd2 line by culturing these parasites in $2 \mu \mathrm{M}$ doxycycline and $200 \mu \mathrm{M}$ IPP for one

428 week. ${ }^{11,55}$ As expected for an apicoplast-targeted protein, the PPS-GFP signal in these parasites

429 displayed a constellation of dispersed fluorescent foci, rather than the concentrated signal observed

430 in untreated parasites (Figure 5D and Figure 5- figure supplement 4). Western blot analysis of the

431 PPS-RFP parasites revealed two bands at the expected molecular weight of the full-length protein

432 and a smaller, proteolytically processed form, consistent with import into the apicoplast lumen

433 (Figure 5E). ${ }^{28}$ In the apicoplast-disrupted parasites, however, only a single PPS-RFP band at the

434 size of the full-length protein was detected, as expected for loss of apicoplast import and lack of

435 protein processing. ${ }^{11,38}$ On the basis of these observations, we conclude that PF3D7_0202700 is

436 an apicoplast-targeted PPS. This localization, the predicted ability of this enzyme to synthesize

437 polyprenyl PPs longer than 4 isoprenes, and our observation that decaprenol rescued FOS-induced

438 defects in apicoplast biogenesis all suggested a critical role for this protein in apicoplast

439 maintenance. 


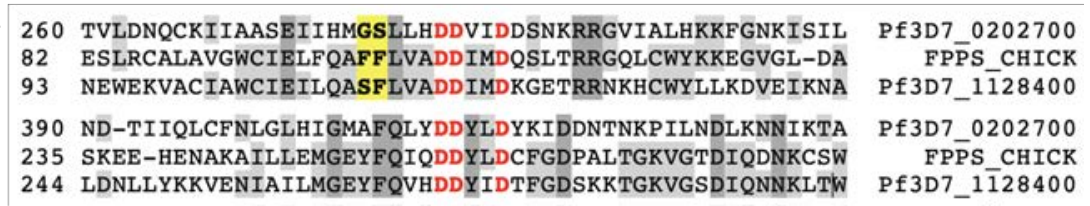

B. Pf3D7_0200700 E. coli OPPS

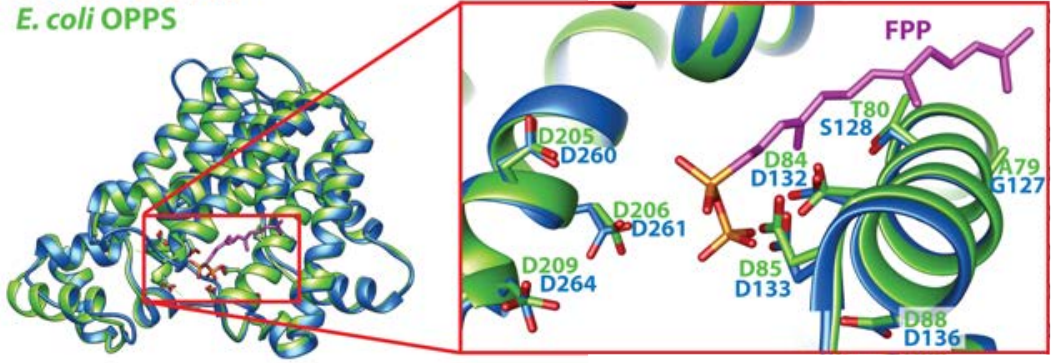

C.

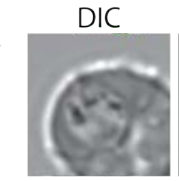

DAPI
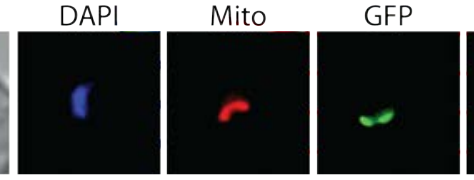

Merge
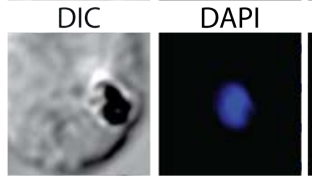

a-ACP
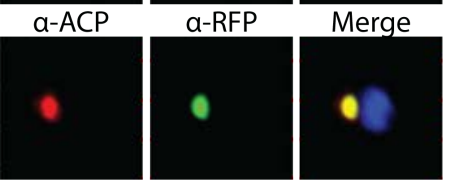

D.

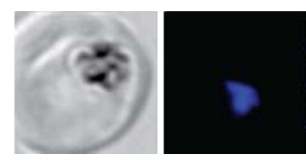

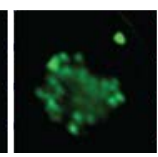
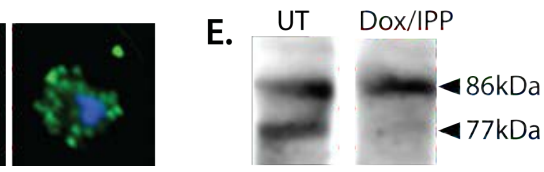

Figure 5. Sequence alignment and localization of the polyprenyl synthase (PPS) PF3D7_0202700 to the apicoplast. (A) Focal sequence alignment of avian farnesyl pyrophosphate synthase (FPPS, Uniprot P08836) with its two P. falciparum homologs reveals the presence of conserved metalbinding DDXXD motifs (red) expected for polyprenyl synthase activity and chain-length determination residues (yellow) upstream of the first DDXXD. (B) Homology model of PF3D7_0202700 using E. coli octaprenyl pyrophosphate synthase (PDB 3WJK) as a structural template. The inset box is an enlargement of the active-site pocket showing the conserved Asp residues, bound FPP substrate, and product length-determining residues just upstream of the first DDXXD motif. (C) Brightfield (BF), fluorescence images (top) of live parasites episomally expressing PPS-GFP and stained with $10 \mathrm{nM}$ Mitotracker Red and (bottom) immunofluorescence analysis (IFA) images of fixed parasites episomally expressing PPS-RFP stained with anti-RFP and anti-apicoplast ACP antibodies. (D) IFA images of fixed parasites expressing PPS-RFP that had been treated for $>7$ days with $2 \mu \mathrm{M}$ doxycycline (Dox) and $200 \mu \mathrm{M}$ IPP (to stably induce apicoplast loss) and stained with anti-RFP antibody to visualize PPS distribution. (E) Western blot analysis of untreated (UT) or Dox/IPP-treated parasites episomally expressing PPS-RFP. PPS-RFP expression was visualized using an anti-RFP antibody. The full western blot image is included in Figure 5- Source Data 1. 
459 Figure supplement 1. Full sequence alignment of PF3D7_0202700, PF3D7_1128400, and avian 460 FPPS (Uniprot P08836).

461 Figure supplement 2. Epifluorescence microscopy images and statistical analysis of PfMev and 462 D10 parasites treated with $10 \mu \mathrm{M}$ MMV091313.

463 Figure supplement 3. Results of sequence-similarity searches for PF3D7_0202700 using NCBI 464 BLAST and MPI HHpred.

465 Figure supplement 4. Additional epifluorescence microscopy images of Dd2 parasite episomally 466 expressing PPS-GFP or PPS-RFP.

467 Source data 1. Uncropped western blot image detecting PPS-RFP expression in parasites.

469 PPS is essential for parasite viability and apicoplast biogenesis. The genomic locus for 470 PF3D7_0202700 was reported to be refractory to disruption in recent genome-wide KO studies in $471 P$. berghei $i^{43}$ and $P$. falciparum, ${ }^{44}$ suggesting an essential function. To directly test its functional 472 essentiality in P. falciparum, we used CRISPR/Cas9 $9^{56}$ to tag the endogenous gene in Dd2 parasites 473 to encode a C-terminal hemagglutinin (HA)-FLAG epitope fusion and the aptamer/TetR-DOZI 474 system $^{57}$ that enables ligand-dependent regulation of protein expression using the non-toxic small 475 molecule, anhydrotetracycline (aTc). In this system, normal protein expression occurs $+\mathrm{aTc}$ and 476 translational repression is induced upon aTc washout (Figure 6A). Correct integration into the 477 genomic locus with the expected genotype in both polyclonal and clonal parasites was confirmed 478 by Southern blot (Figure 6- figure supplement 1). Expression of the $\sim 60 \mathrm{kDa}$ HA-FLAG-tagged, 479 endogenous mature protein was detected by western blot (Figure 6B).

481 (KD) parasites and monitored their growth $\pm \mathrm{aTc}$ over multiple intraerythrocytic lifecycles.

482 Because of inconsistency in detecting the endogenous PPS by western blot (WB), possibly due to 483 low protein expression, we monitored PPS transcript levels by RT-qPCR in lieu of WB analysis. 484 We observed robust knockdown of PPS mRNA levels by the second intraerythrocytic cycle 485 (Figure 6C). The fate of target mRNA in the aptamer/TetR-DOZI system has not been 
486 characterized in depth. Our data is consistent with a prior report ${ }^{58}$ and suggests that TetR-DOZI

487 binding after aTc washout leads to mRNA transcript degradation, possibly within stress granules

488 targeted by DOZI-bound transcripts. ${ }^{57}$ In the presence of aTc, culture parasitemia expanded in a

489 step-wise fashion over the $\sim 10$ days of the growth assay such that the culture needed to be split

490 multiple times to avoid over-growth (Figure 6C). Without aTc, however, the culture grew normally

491 over the first 3 intraerythrocytic cycles but showed a major growth defect in the fourth cycle

492 consistent with extensive parasite death observed by blood smear (Figure 6C and Figure 6- figure

493 supplements 1 and 2). Parasite growth under -aTc conditions was rescued in the presence of 200

$494 \mu \mathrm{M}$ exogenous IPP (Figure 6C), indicating an essential PPS function within the apicoplast.

To test a role for PPS in synthesizing polyprenyl PP groups, we attempted to rescue parasite

496 growth in -aTc conditions by adding $5 \mu \mathrm{M} \mathrm{FOH,} \mathrm{GGOH,} \mathrm{or} \mathrm{decaprenol.} \mathrm{We} \mathrm{observed} \mathrm{that} \mathrm{only}$

497 decaprenol, but not $\mathrm{FOH}$ or GGOH, rescued parasite growth -aTc, and the magnitude of rescue by

498 decaprenol was comparable to IPP (Figure 6C and Figure 6- figure supplement 2). These

499 observations strongly suggest that PPS has an essential function downstream of IPP synthesis in

500 converting isoprenoid precursors into longer-chain linear polyprenyl-PPs containing at least 5-10

501 isoprene units. 

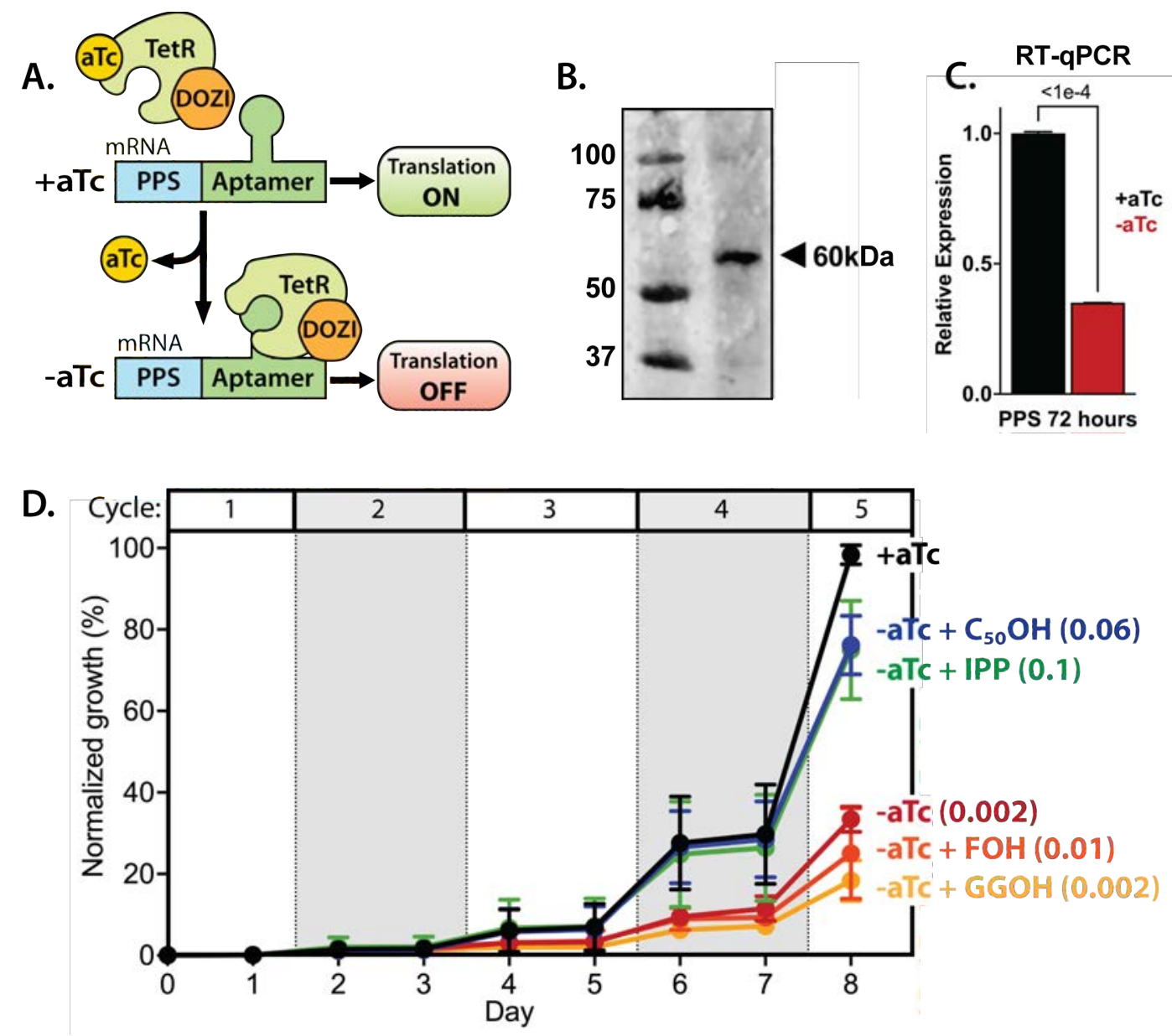

Figure 6. PPS (PF3D7_0202700) is essential for parasite viability and apicoplast function. (A) Schematic depiction of the aptamer/TetR-DOZI system for ligand-dependent protein expression. The target protein (PPS) is translated normally in the presence of anhydrotetracycline (aTc) but its translation is repressed without aTc. (B) Western blot of endogenously tagged PPS-HA/FLAG and probed with anti-HA epitope antibody showing detection of tagged PPS at the expected size for mature PPS of $\sim 60 \mathrm{kDa}$. (C) RT-qPCR analysis of PPS transcript levels (normalized to the average of two nuclear control genes) in synchronous parasites cultured for 72 hours \pm aTc. Significance of the observed difference was evaluated by two-tailed unpaired t-test with the indicated P value. (D) Synchronous growth assay of Dd2 parasites tagged at the PPS locus with the aptamer/TetR-DOZI system and grown $\pm \mathrm{aTc}$ and $\pm 200 \mu \mathrm{M}$ IPP or $5 \mu \mathrm{M}$ farnesol $(\mathrm{FOH})$, geranylgeraniol $(\mathrm{GGOH})$, or decaprenol $\left(\mathrm{C}_{50}-\mathrm{OH}\right)$. Parasitemia values for each condition are the average $\pm \mathrm{SD}$ of two biological replicates. Two-tailed unpaired t-test analysis was used to determine the significance of observed parasitemia differences on day 8 for each condition compared to parasites cultured $+\mathrm{aTc}$ (P values given in parentheses).

Figure supplement 1. Scheme for modification of the PPS genomic locus to integrate the aptamer/TetR-DOZI system and Southern blot confirming correct integration.

520 Figure supplement 2. Blood-smear images of Dd2 parasites tagged at the PPS locus with the aptamer/TetR-DOZI system and grown \pm aTc for 8 days. 
To test if PPS function is required for apicoplast biogenesis, we cultured PPS KD parasites

525 in + aTc or -aTc/+IPP conditions for 12 days and then assessed apicoplast morphology in fixed

526 parasites by $\alpha \mathrm{ACP}$ immunofluorescence. We reasoned that IPP would rescue parasite viability

527 upon PPS KD but not interfere with assessing any defect in apicoplast biogenesis if IPP synthesis

528 is upstream of PPS function. Immunofluorescence analysis (IFA) revealed that $\sim 30 \%$ of parasites

529 cultured in -aTc conditions had a dispersed ACP signal indicative of apicoplast disruption (Figure

530 7- figure supplement 1). Although this observation supports a critical role for PPS in apicoplast

531 biogenesis, we wondered why PPS KD did not result in a higher fraction of parasites with disrupted

532 apicoplast. We hypothesized that residual PPS expression resulting from incomplete KD combined

533 with high IPP levels due to culture supplementation and endogenous MEP pathway activity might

534 enable sufficient synthesis of polyprenols to attenuate the impact of PPS KD on apicoplast

535 biogenesis.

To test this hypothesis and the contribution of MEP activity to the observed phenotype, we

537 synchronized parasites to the ring stage and cultured them in \pm aTc conditions for 96 hours (two

538 48-hour growth cycles) to knock down PPS expression before adding FOS and IPP at the start of

539 the third growth cycle (Figure 7A). In this experiment, FOS was expected to inhibit endogenous

540 MEP pathway activity without impacting apicoplast biogenesis since it was added concurrently

541 with IPP, which fully rescues parasites from growth and apicoplast defects induced by FOS (Figure

542 1). ${ }^{11,21}$ We first used IFA to assess apicoplast morphology in schizonts at the end of the third

543 growth cycle (38 hours after adding FOS and IPP). We observed normal apicoplast elongation in

$544+$ aTc parasites but focal, unbranched apicoplast morphology in the vast majority ( $>80 \%)$ of -aTc

545 parasites (Figure 7, B and C, and Figure 7- figure supplement 2). Substitution of IPP with FOH or

546 GGOH resulted in a nearly identical apicoplast elongation defect in -aTc parasites. In contrast, 
547 substituting IPP with decaprenol resulted in normal apicoplast elongation in both $+\mathrm{aTc}$ and -aTc

548 parasites (Figure 7, B and C, and Figure 7- figure supplement 2). The selective ability of

549 decaprenol to rescue apicoplast-branching defects in -aTc conditions strongly supports an essential

550 role for PPS in synthesizing long-chain polyprenyl isoprenoids required for apicoplast biogenesis.

551 To further test this conclusion, we maintained parasites in $\pm \mathrm{aTc}$ conditions with FOS and

552 IPP for two additional growth cycles (total of five 48-hour cycles, Figure 7A). Parasites cultured

$553+$ aTc displayed normal elongated apicoplast morphology. In contrast, the -aTc (+FOS and IPP)

554 culture predominantly contained parasites with a dispersed ACP signal indicative of apicoplast

555 loss (Figure 7, D and E, and Figure 7- figure supplement 3). These -aTc parasites also contained a

556 strongly reduced qPCR signal for apicoplast genomic DNA, relative to +aTc parasites (Figure 7F).

557 These results indicate that PPS is essential for apicoplast maintenance and inheritance by daughter

558 parasites such that loss of PPS function (with IPP supplementation) results in parasite progeny

559 lacking the intact organelle. This essential PPS function downstream of IPP synthesis by the MEP

560 pathway is sufficient to explain our observation that blocking pathway activity by FOS or $\triangle \mathrm{DXS}$

561 (Figure 1) inhibits apicoplast biogenesis. 


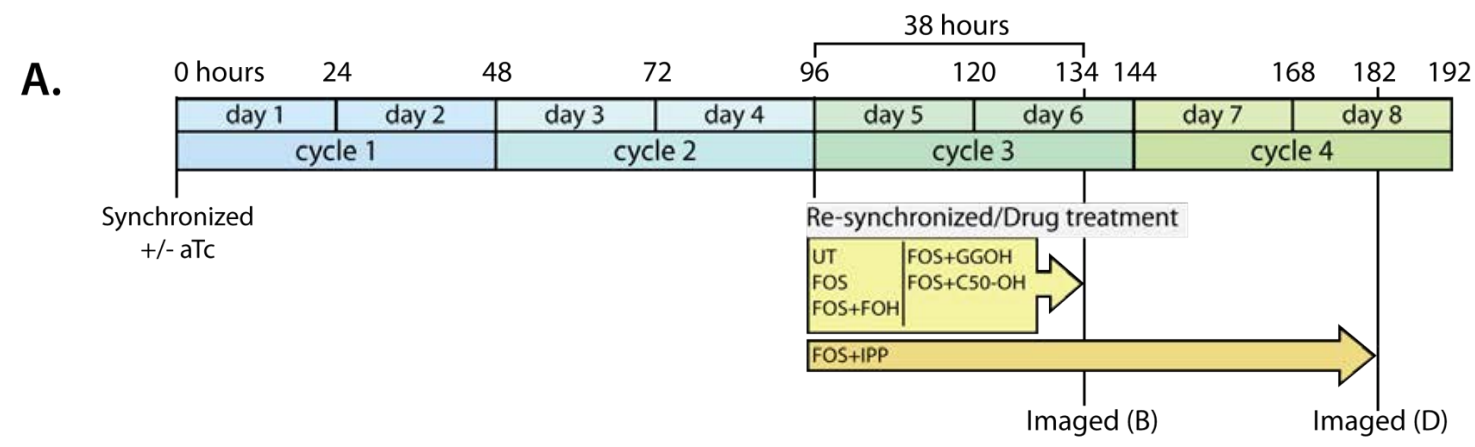

B.
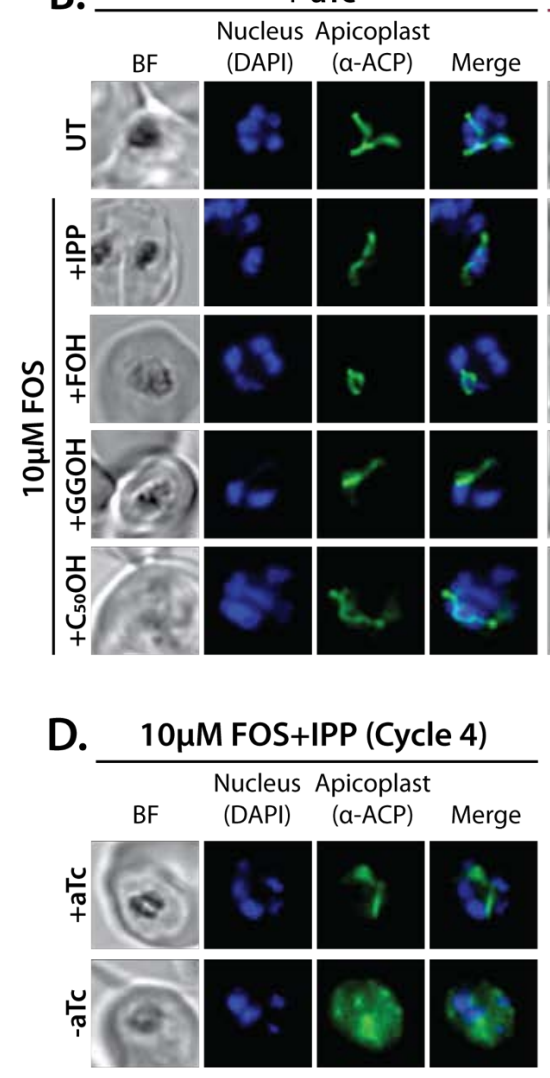

562

563

564

565

566

567

568

569

570

571

572

573

574
- aTc

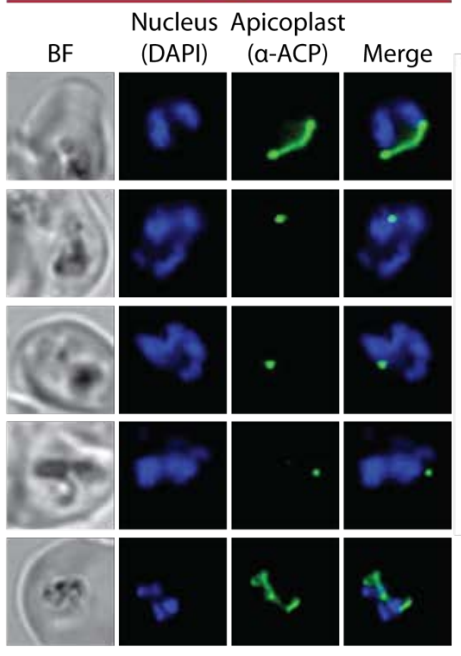

E. $10 \mu \mathrm{M}$ FOS+IPP (Cycle 4) Apicoplast Status

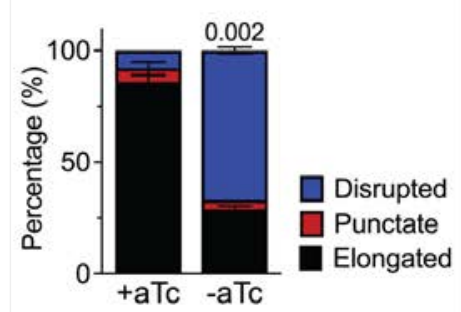

C. PPS-KD $+10 \mu \mathrm{M}$ FOS Apicoplast Status

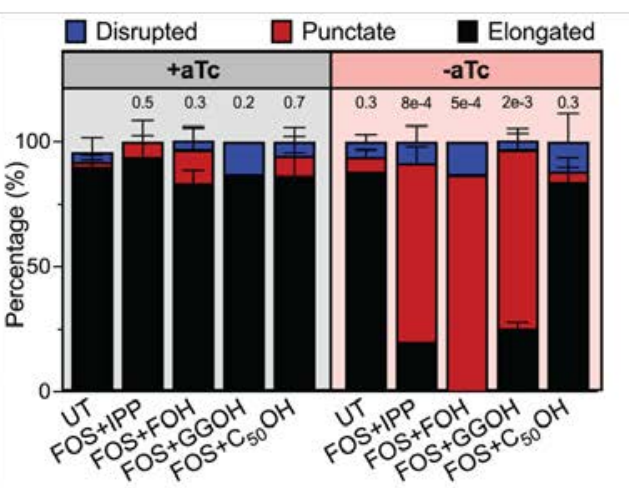

F. $10 \mu \mathrm{M}$ FOS+IPP (Cycle 4) qPCR

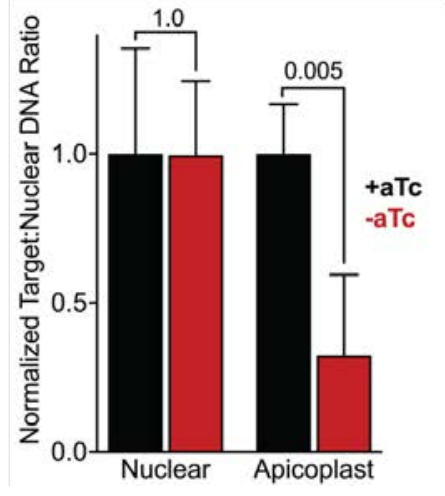

Figure 7. PPS is required for apicoplast biogenesis. (A) Scheme summarizing growth of synchronized PPS knockdown parasites cultured $\pm \mathrm{aTc}$, re-synchronized and treated with $10 \mu \mathrm{M}$ FOS $\pm 200 \mu \mathrm{M}$ IPP or $5 \mu \mathrm{M}$ farnesol $(\mathrm{FOH})$, geranylgeraniol $(\mathrm{GGOH})$, or decaprenol $\left(\mathrm{C}_{50}-\mathrm{OH}\right)$ at 96 hours after initial synchronization, and imaged at 134 and 182 hours after initial synchronization. (B) Immunofluorescence analysis (IFA) of PPS knockdown parasites cultured as described in panel A and imaged at 134 hours (day 6) after initial synchronization to assess apicoplast morphology $\pm \mathrm{aTc}$. (C) Statistical analysis of apicoplast morphology for 50 total parasites imaged for each condition in panel A from two independent experiments. Apicoplast morphologies were scored as punctate (focal), elongated, or disrupted (dispersed); counted; and plotted by histogram as the fractional population with the indicated morphology. Error bars represent standard deviations from replicate experiments. Two-tailed unpaired t-test analysis was used to determine the significance of observed population differences compared to untreated (UT) 
575 parasites (P values given above each condition). (D) Immunofluorescence analysis of PPS

576 knockdown parasites cultured as described in panel A and imaged at 182 hours (day 8) after initial 577 synchronization to assess apicoplast morphology \pm aTc. (E) Statistical analysis of apicoplast 578 morphology for 50 total parasites imaged for each condition in panel D and analyzed as in panel 579 C. (F) Quantitative PCR analysis of the apicoplast:nuclear (Api:Nu) genome ratio for parasites 580 cultured $\pm \mathrm{aTc}$ and imaged in panel D, based on amplification of apicoplast TufA 581 (PF3D7_API02900) or nuclear ADSL (PF3D7_0206700) relative to nuclear I5P 582 (PF3D7_0802500) genes. Indicated qPCR ratios were normalized to +aTc in each case and are the 583 average \pm SD of two biological replicates. Significance of \pm aTc differences was analyzed by two584 tailed unpaired t-test to determine the stated $\mathrm{P}$ value. All parasite samples collected for IFA were 585 imaged by brightfield (BF) and epifluorescence microscopy, with visualization of parasite nuclei 586 by DAPI staining and apicoplast by an anti-apicoplast ACP antibody.

Figure supplement 1. IFA images and analysis of apicoplast morphology in PPS knockdown parasites grown $+\mathrm{aTc}$ or $-\mathrm{aTc} /+\mathrm{IPP}(200 \mu \mathrm{M})$ for 12 days.

590 Figure supplement 2. Additional IFA images of PPS knockdown parasites treated as in Figure $5917 \mathrm{~B}$.

Figure supplement 3. Additional IFA images of PPS knockdown parasites treated as in Figure

No evidence for PPS function in carotenoid synthesis. Despite its strong sequence similarity to

known polyprenyl synthases that catalyze the head-to-tail condensation of isoprenoid precursors,

PF3D7_0202700 has also been proposed to catalyze the biochemically distinct head-to-head condensation of 20-carbon GGPP groups into 40-carbon phytoene and thus function as a phytoene synthase (PSY) within a broader pathway of carotenoid biosynthesis proposed to exist in Plasmodium parasites (Figure 8A). ${ }^{53,} 59$ Polyprenyl synthases and phytoene synthases are mechanistically distinct enzymes that lack significant sequence similarity but are thought to share a common isoprenoid-related protein fold that reflects their ancient divergence from a common ancestral enzyme. ${ }^{47,}{ }^{60}$ Given the mechanistic differences between head-to-tail and head-to-head condensation of isoprenoids (Figure 8A), which involve distinct positioning of substrate pyrophosphate groups within each active site, there is no known enzyme that is capable of 
608 is without biochemical precedent. Nevertheless, we considered whether this protein might also

609 have PSY function and evaluated whether existing observations supported or contradicted a

610 proposed role for this protein in carotenoid biosynthesis.

611

As noted previously, untargeted sequence similarity searches via NCBI BLAST ${ }^{51}$ and MPI

612 HHpred ${ }^{52}$ with PF3D7_0202700 as the query sequence only identify polyprenyl synthase

613 homologs from bacteria, algae, and plants (Figure 5- figure supplement 3) and fail to identify PSY

614 homologs. Furthermore, targeted pairwise alignments show no evidence of significant sequence

615 homology between PF3D7_0202700 and confirmed eukaryotic or prokaryotic PSY sequences

616 from Arabidopsis thaliana (Uniprot P37271, chloroplast-targeted) ${ }^{61}$ or Erwinia herbicola

617 (Pantoea agglomerans, Uniprot D5KXJ0), ${ }^{62}$ respectively. Finally, the prior proposal of PSY

618 activity by PF3D7_0202700 was based in part on its sequence similarity to an annotated PSY from

619 Rubrivivax gelatinosus bacteria (NCBI accession BAA94032) that also appeared to contain

620 sequence features expected of a head-to-tail polyprenyl synthase. ${ }^{53}$ We noted that the functional

621 annotation of this bacterial protein was subsequently revised to a geranylgeranyl-PP synthase

622 (Uniprot I0HUM5), ${ }^{63}$ thus explaining its sequence similarity to PF3D7_0202700 and the

623 homology of both proteins to known polyprenyl synthases. On the basis of these sequence

624 analyses, we considered it unlikely that PF3D7_0202700 had dual activity as a PSY.

625 The prior work studied the antiparasitic effects of the squalene synthase inhibitor, zaragozic

626 acid (ZA, also called squalestatin), that inhibited blood-stage $P$. falciparum growth (EC $\left.\mathrm{E}_{50} \sim 5 \mu \mathrm{M}\right)$

627 and was proposed to specifically target PF3D7_0202700 based on observation of a $\sim 6$-fold

628 increase in $\mathrm{EC}_{50}$ for parasites episomally expressing a second copy of this protein. ${ }^{59}$ We repeated

629 these experiments in Dd2 parasites and observed a similar $\mathrm{EC}_{50}$ of $\sim 10 \mu \mathrm{M}$ for ZA that increased

6305 -fold to $\sim 50 \mu \mathrm{M}$ in Dd2 parasites episomally expressing PPS-RFP (Figure 8- figure supplement 
631 1). However, in contrast to PPS knockdown (Figure 6D), lethal growth inhibition by ZA was not

632 rescued by exogenous IPP (Figure 8- figure supplement 1) and did not affect apicoplast elongation

633 (Figure 8- figure supplement 2). These contrasting phenotypes strongly suggest that PPS, and more

634 broadly the apicoplast, are not uniquely targeted by ZA. The basis for why PPS over-expression

635 reduces parasite sensitivity to $\mathrm{ZA}$ is unclear but may reflect drug interactions with broader

636 isoprenoid metabolism outside the apicoplast that are rescued, directly or indirectly, by reaction

637 products of PPS.

$\beta$-carotene, a 40-carbon carotenoid derived from phytoene, was previously detected by

639 mass spectrometry in extracts of $P$. falciparum-infected erythrocytes and suggested to be

640 biosynthesized by parasites based on the lack of detection in extracts of uninfected erythrocytes. ${ }^{53}$

641 Using our PPS knockdown line, we tested whether translational repression of PF3D7_0202700

642 impacted detectable levels of $\beta$-carotene in parasites, as predicted to occur if PPS also functioned

643 as a PSY. After synchronization, PPS knockdown parasites were grown \pm aTc for 120 hours and

644 harvested at the end of the third intraerythrocytic growth cycle, which immediately precedes the

645 growth defect observed in Figure 6D. Saponin pellets of these parasites were extracted in acetone

646 and analyzed by liquid chromatography/tandem mass spectrometry for $\beta$-carotene (Figure 8 - figure

647 supplement 3). We observed indistinguishable low levels of $\beta$-carotene in both samples (Figure

648 8B), providing no evidence that PPS plays a role in carotenoid biosynthesis.

Although uninfected erythrocytes washed in AlbuMAX-free RPMI lacked detectable $\beta$ -

650 carotene, we observed that extracts of uninfected erythrocytes incubated in complete RPMI

651 medium containing AlbuMAX I had $\beta$-carotene levels that were nearly identical to extracts of

652 parasite-infected erythrocytes (Figure 8B). Analysis of AlbuMAX (ThermoFisher catalog

$653 \# 11020021)$ by mass spectrometry revealed modest levels of $\beta$-carotene (Figure 8- figure 
654 supplement 4), consistent with the bovine origin of AlbuMAX (lipid-rich bovine serum albumin)

655 and the plant-based diet of these animals expected to contain $\beta$-carotene. These results are

656 sufficient to explain the presence of carotenoids like $\beta$-carotene in parasite extracts. In summary,

657 we find no evidence that PPS function contributes to $\beta$-carotene levels in $P$. falciparum-infected

658 erythrocytes, which we suggest non-specifically take up exogenous plant-derived $\beta$-carotene

659 associated with AlbuMAX in the culture medium.

661 into biosynthetic products that had reverse-phase HPLC retention times similar to all-trans-lutein

662 or $\beta$-carotene standards, suggesting de novo synthesis of these isoprenoid products. ${ }^{53}$ Because the

663 extracted products were radioactive, their identity could not be directly confirmed by tandem mass

664 spectrometry. To directly test if blood-stage $P$. falciparum parasites incorporate isoprenoid

665 precursors into $\beta$-carotene, as predicted for active biosynthesis, we cultured the PfMev parasites

666 in $50 \mu \mathrm{M}$ of $2{ }^{13} \mathrm{C}$-mevalonate in the presence of $10 \mu \mathrm{M}$ FOS. This strategy was chosen to inhibit

667 MEP pathway activity, ensure full ${ }^{13} \mathrm{C}$-labeling of the endogenous IPP and DMAPP precursor pool

668 produced by the cytoplasmic bypass enzymes, and result in a distinguishable 8 Da mass increase

669 for any $\beta$-carotene derived from de novo synthesis. We previously showed that this strategy results

670 in complete ${ }^{13} \mathrm{C}$-labeling of endogenous IPP and FPP. ${ }^{29}$ Parasites were expanded to high

671 parasitemia over several days under ${ }^{13} \mathrm{C}$-labeling conditions before extraction and analysis by mass

672 spectrometry. Although we readily detected unlabeled $\beta$-carotene ( $\mathrm{m} / \mathrm{z} 536.4)$, which we attribute

673 to culture medium AlbuMAX, we were unable to detect ${ }^{13} \mathrm{C}$-labeled $\beta$-carotene $(\mathrm{m} / \mathrm{z} 544.4)$ (Figure

$6748 \mathrm{C})$. In contrast to the parasite analysis, we readily detected fully ${ }^{13} \mathrm{C}$-labeled $\beta$-carotene $(\mathrm{m} / \mathrm{z}$

675 576.6) produced by E. coli bacteria engineered to biosynthesize $\beta$-carotene ${ }^{64}$ and grown in minimal

676 M9 medium with uniformly labeled ${ }^{13} \mathrm{C}$-glucose as the sole carbon source (Figure 8D). In 
680 apicoplast biogenesis.
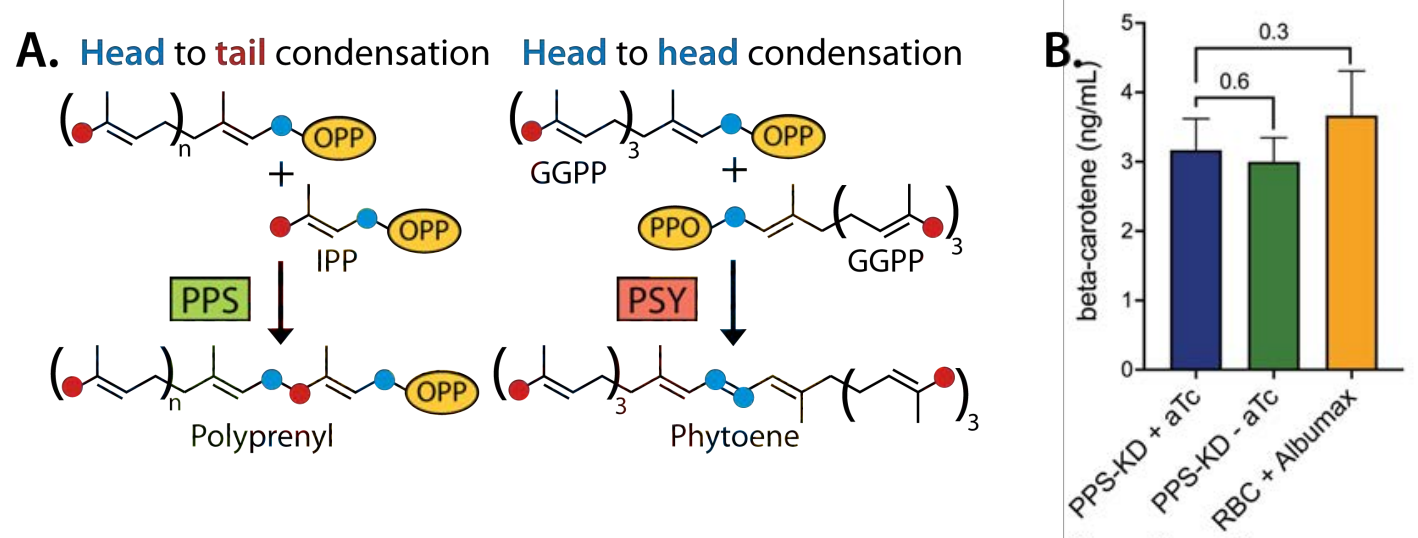

C.

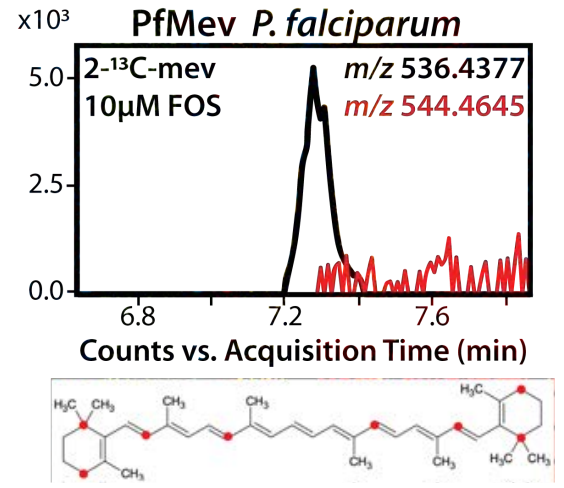

681

682

683

684

685

686

687

688

689

690

691

692

693
D.

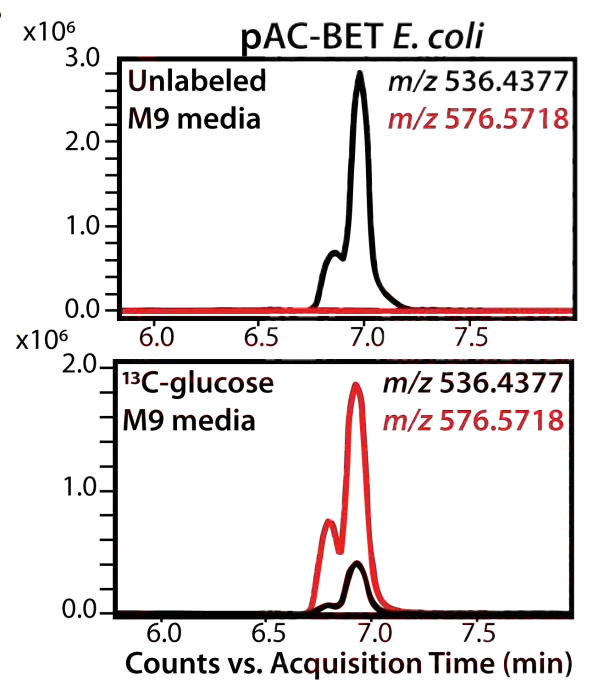

Figure 8. No evidence that PPS contributes to carotenoid synthesis by P. falciparum. (A) Schematic depiction of head-to-tail arrangement of prenyl groups during polyprenyl-PP synthesis versus head-to-head arrangement of geranylgeranyl-PP groups during phytoene synthesis. (B) Mass spectrometry determination of unlabeled $\beta$-carotene levels in PPS knockdown parasites grown for 6 days \pm aTc or in uninfected red blood cells incubated in complete media containing Albumax. Measured $\beta$-carotene levels are the average \pm SD of 3 biological replicates, whose differences were analyzed by two-tailed unpaired t-test for significance ( $\mathrm{P}$ values given relative to + aTc sample). (C) Intensity versus retention time plot for liquid chromatography-mass spectrometry determination of unlabeled and ${ }^{13} \mathrm{C}$-labeled $\beta$-carotene in NF54 PfMev parasites cultured for 6 days in $50 \mu \mathrm{M} 2-{ }^{13} \mathrm{C}$-mevalonate and $10 \mu \mathrm{M}$ fosmidomycin. Below: schematic depiction of the 8 carbon atoms in $\beta$-carotene expected to be labeled with ${ }^{13} \mathrm{C}$ for synthesis from 
IPP derived from $2-{ }^{13} \mathrm{C}$-mevalonate in PfMev parasites. (D) Intensity versus retention time plot for liquid chromatography-mass spectrometry determination of unlabeled and ${ }^{13} \mathrm{C}$-labeled $\beta$-carotene in pAC-BETAipi E. coli grown in unlabeled or fully ${ }^{13} \mathrm{C}$-labeled glucose as the sole carbon source in M9 minimal media. The two peaks reflect the presence of an isomeric mix of all-trans and cis $\beta$-carotene produced by the pAC-BETAipi E. coli, as previously reported. ${ }^{64}$

Figure supplement 1. 48-hour growth inhibition curves for treatment of Dd2 parasites with

701 zaragozic acid without or with episomal expression of PPS-RFP or $200 \mu \mathrm{M}$ IPP.

Figure supplement 2. Epifluorescence microscopy images of D10 parasites treated with $160 \mu \mathrm{M}$ multinuclear schizonts.

Figure supplement 3. Fragment ion spectrum for unlabeled beta-carotene determined by tandem mass spectrometry of $\beta$-carotene commercial standard.

707 Figure supplement 4. Intensity versus retention time plot for liquid chromatography-mass

\section{DISCUSSION}

Biosynthesis of the isoprenoid precursors, IPP and DMAPP, is a well-established essential

712 function of the Plasmodium apicoplast, but prior work has focused nearly exclusively on the

713 critical roles of isoprenoids for diverse cellular processes outside this organelle. ${ }^{7,11,15,20}$ We have

714 elucidated a novel arm of isoprenoid metabolism within the apicoplast that is required for

715 biogenesis of this critical organelle (Figure 9). This discovery expands the paradigm for isoprenoid

716 utilization by malaria parasites, uncovers a novel essential feature of apicoplast biology, and

717 identifies a key enzyme in this pathway suitable for development as a therapeutic target.

Implications for general understanding of apicoplast functions. Our study, which was inspired

720 by prior hints in the literature, ${ }^{25-27}$ firmly establishes a novel essential role for MEP pathway

721 activity in supporting apicoplast biogenesis, in addition to its recognized role producing IPP

722 required outside this organelle. The dual roles of this pathway in both apicoplast-specific and

723 broader parasite cellular biology provide a clear exception to the prevailing binary model of blood- 
724 stage apicoplast metabolism that pathway functions in this organelle can be cleanly segregated into

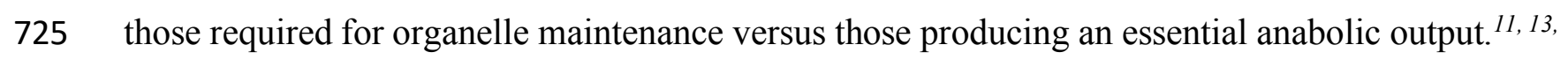

726 15, 65 Thus, IPP synthesis by the MEP pathway requires apicoplast maintenance, which in turn

727 depends on IPP synthesis. The two processes are convoluted and interdependent.

728 Our results also support the emerging paradigm ${ }^{12,16-18}$ that inhibition of apicoplast

729 maintenance pathways can kill parasites with first-cycle kinetics that defy the delayed-death

730 phenotype commonly observed for translation-blocking antibiotics such as doxycycline that target

731 organelle housekeeping. ${ }^{6,12}$ Indeed, blocking IPP synthesis causes same-cycle defects in apicoplast

732 biogenesis, which are expected to produce non-viable parasite progeny independent of lethal

733 dysfunctions in isoprenoid-dependent metabolism outside the organelle. Analysis of the timing of

734 FOS-induced defects in apicoplast branching also provides an unexpected and incisive window

735 into the differential compartmentalization of IPP essentiality in parasites. We observed that FOS-

736 treated parasites display apicoplast-elongation defects in early schizogony but continue to divide

737 nuclear DNA and transition into mature schizonts before stalling prior to segmentation (Figure

738 1A). Thus, the critical role of IPP for apicoplast biogenesis precedes the broader cellular need for

739 IPP outside the organelle in mature schizonts, suggested by recent works to predominantly reflect

740 essential roles for IPP-dependent protein prenylation. ${ }^{15,30}$ Although MEP pathway activity begins

741 in ring-stage parasites, ${ }^{31,32}$ we observed identical inhibition of apicoplast elongation in schizonts

742 independent of whether FOS was added to rings concomitant with synchronization or to

743 trophozoites 12 hours after synchronization (Figure 1A). This observation suggests that IPP

744 utilization in the apicoplast depends on de novo synthesis rather than a pre-existing metabolite

745 pool, possibly because IPP does not accumulate in the apicoplast and/or that IPP synthesis within

746 the organelle is differentially partitioned for export and internal utilization. 
748 Why does apicoplast biogenesis depend on IPP synthesis? The essential function of PPS in

749 apicoplast maintenance is sufficient to explain the apicoplast reliance on IPP synthesis unveiled

750 by FOS treatment of parasites. Although the dominant polyprenyl-PP product of apicoplast PPS

751 in parasites remains uncertain, sequence features, prior in vitro enzymology, and the ability of

752 exogenous decaprenol but not GGOH or FOH to rescue PPS knockdown indicate that linear

753 polyprenyl-PP products longer than 4 and as long as 10 isoprene units are critical for apicoplast

754 maintenance.${ }^{24}$ Prior work suggested a dual function for PF3D7_0202700 as a phytoene synthase

755 (PSY) that also condenses isoprenoid precursors, ${ }^{53,59}$ but we found no evidence to support this

756 proposed PSY function or carotenoid biosynthesis more broadly. Synthesis of octaprenyl-PP by

757 PF3D7_0202700 was previously proposed to be critical for ubiquinone biosynthesis in the parasite

758 mitochondrion. ${ }^{24}$ Localization of this protein to the apicoplast (Figure 5) and observation that

759 exogenous IPP rescues the grown defects of its knockdown (Figure 6D) strongly suggest that its

760 activity is not required for mitochondrial ubiquinone biosynthesis and that its essential function is

761 specific to the apicoplast.

762 Plant chloroplasts synthesize linear polyprenyl isoprenoids to serve a wide variety of

763 functions that are only partially understood but include key roles in light harvesting and

764 photosynthesis, oxidative stress protection, and as precursors of signaling and defense molecules

765 that function outside the chloroplast (e.g., abscisic acid, gibberellins, and terpenes). ${ }^{66-68}$ The

766 Plasmodium apicoplast has lost photosynthesis capabilities and has uncertain carotenoid and

767 terpene synthesis capacity. Volatile terpenes ${ }^{69}$ and carotenoids ${ }^{53}$ have been detected in $P$.

768 falciparum-infected erythrocytes, but the parasite genome lacks enzyme homologs of the relevant

769 synthases required for terpene and carotenoid biosynthesis. ${ }^{1,7}$ Furthermore, we found no evidence 
770 of de novo $\beta$-carotene synthesis by parasites, and results herein as well as recent studies ${ }^{70,71}$

771 indicate that these metabolites can derive from erythrocyte and/or culture medium sources rather

772 than parasite-specific synthesis. Thus, these known functions in chloroplasts seem uncertain or

773 unlikely to explain apicoplast reliance on longer-chain polyprenyl synthase activity in malaria

774 parasites. Longer-chain polyprenyl-PPs and related dolichols serve as membrane-bound glycan

775 carriers for protein glycosylation, but these activities in Plasmodium appear to occur in the

776 endoplasmic reticulum as they do in other organisms. ${ }^{20,72,73}$

777 Linear polyprenyl alcohols have been found to be important components of plant

778 membranes, especially chloroplast membranes, where they are proposed to modulate membrane

779 structure, fluidity, and dynamics. ${ }^{66,68,74,75}$ In the absence of other known roles for longer-chain

780 polyprenyl-PPs in the apicoplast, we hypothesize that linear polyprenols or polyprenyl phosphates

781 may serve as critical components of the apicoplast membranes and be required for maintaining

782 membrane fluidity during organelle biogenesis (Figure 9). A prior mass spectrometry-based

783 lipidomics study of isolated apicoplasts focused primarily on the fatty acid and phospholipid

784 composition of this organelle and did not characterize isoprenoid components. ${ }^{76}$ Selective isotopic

785 labeling of parasite-synthesized isoprenoids by $2-{ }^{13} \mathrm{C}-\mathrm{mevalonate}$ in the PfMev line, combined

786 with apicoplast isolation and our PPS knockdown line, can potentially identify specific apicoplast

787 isoprenoids whose synthesis depends on PPS activity and thus clarify why apicoplast biogenesis

788 requires longer-chain polyprenyl synthase activity.

789 Independent of its specific role in apicoplast biogenesis, PPS function is critical for parasite

790 survival and thus constitutes a new essential arm of isoprenoid metabolism in the apicoplast

791 suitable for development as a therapeutic target. BLAST analysis of the human genome using the

792 PPS protein sequence as query reveals a variety of polyprenyl synthase homologs with modest 20- 
$30 \%$ sequence identity to $25-50 \%$ of the PPS sequence. The substantial sequence differences with

human orthologs will facilitate selective targeting of PPS by chemical inhibitors. Identification of

PPS as an apicoplast-targeted enzyme indicates that new metabolic pathways and functions remain

to be discovered and/or localized to the apicoplast. These novel functions, which are predicted to

797 be required for organelle maintenance, will enhance our understanding of fundamental apicoplast

biology and provide new candidate drug targets for antimalarial therapies.

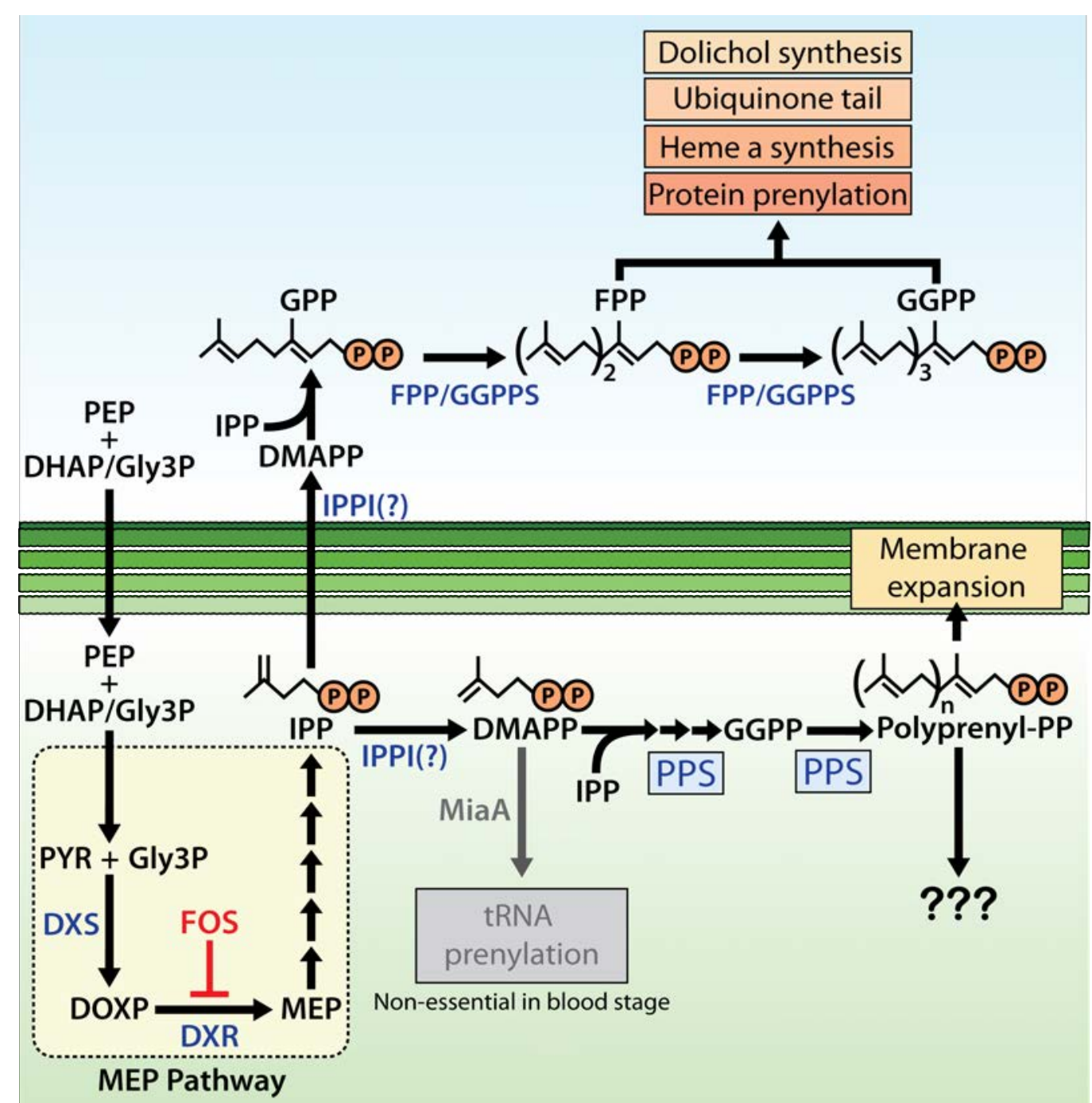

Figure 9. Schematic diagram of apicoplast isoprenoid metabolism in blood-stage $P$. falciparum parasites. $\mathrm{PK}=$ pyruvate kinase II, TPI $=$ triose phosphate isomerase, IPPI = IPP isomerase, $\mathrm{PEP}$ $=$ phosphoenolpyruvate, DHAP $=$ dihydroxyacetone phosphate, $\mathrm{PYR}=$ pyruvate, Gly3P $=$ glyceraldehyde-3-phosphate. Question marks indicate uncertainty in the identity of the proposed IPP isomerase and in the role of polyprenyl isoprenoid products of PPS in apicoplast biogenesis. 
MATERIALS AND METHODS

807 Materials: All reagents were of the highest purity commercially available. The vendor and catalog

808 number are given for individual compounds when first mentioned.

Fluorescence Microscopy: For live-cell experiments, parasite samples were collected at 38 hours

811 after synchronization with 5\% D-sorbitol (Sigma S7900). Parasite nuclei were visualized by

812 incubating samples with 1-2 $\mu \mathrm{g} / \mathrm{ml}$ Hoechst 33342 (Thermo Scientific Pierce 62249) for 10-20

813 minutes at room temperature. The parasite apicoplast was visualized in D10 28 or NF54

814 mevalonate-bypass ${ }^{29}$ cells using the $\mathrm{ACP}_{\mathrm{L}}$-GFP expressed by both lines. The parasite

815 mitochondrion was visualized by incubating parasites with $10 \mathrm{nM}$ MitoTracker Red CMXROS

816 (Invitrogen Life Technologies M7512) for 15 minutes prior to wash-out and imaging. For

817 immunofluorescence assay (IFA) experiments, parasites were fixed, stained, and mounted as

818 previously described. ${ }^{77}$ For IFA images, the parasite apicoplast was visualized using a polyclonal

819 rabbit anti-ACP antibody ${ }^{78}$ and an anti-rabbit fluorescent $2^{\circ}$ antibody, the nucleus was stained with

820 ProLong Gold Antifade Mountant with DAPI (Invitrogen Life Technologies P36931), and PPS-

821 GFP was visualized with a Goat anti-GFP antibody (Abcam ab5450). Images were taken on

822 DIC/brightfield, DAPI, GFP, and RFP channels using either a Zeiss Axio Imager or an EVOS

823 M5000 imaging system. Fiji/ImageJ was used to process and analyze images. All image

824 adjustments, including contrast and brightness, were made on a linear scale.

825 For phenotypic analyses, apicoplast morphologies for each experimental condition were

826 assessed for 25 parasites in each of two biological replicate experiments (50 parasites total per

827 condition). Apicoplast morphologies were scored as elongated, focal, or dispersed; counted; and

828 plotted by histogram as the fractional population with the indicated morphology. Statistical 
829 significance of observed differences from untreated parasites was assessed in GraphPad Prism 9

830 by two-tailed unpaired t test. $\mathrm{P}$ values were rounded to one significant figure, and non-significance

831 was concluded for differences with $\mathrm{P}$ values $>0.05$.

832

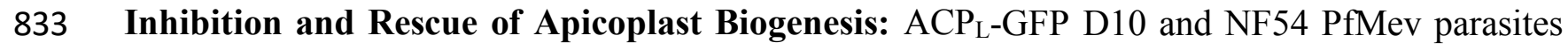

834 were synchronized with 5\% (w/v) D-sorbitol for 10 minutes at room temperature and returned to 835 culture in $10 \mu \mathrm{M}$ fosmidomycin (Invitrogen Life Technologies F23103), $100 \mathrm{nM}$ atovaquone 836 (Caymen Chemicals 23802), $2 \mu \mathrm{M}$ DSM1, ${ }^{79} 6 \mu \mathrm{M}$ blasticidin-S (Invitrogen Life Technologies 837 R21001), 5 nM WR99210 (Jacobus Pharmaceuticals), $160 \mu \mathrm{M}$ zaragozic acid/squalestatin 838 (Caymen Chemicals 17452), or $2 \mu \mathrm{M}$ MMV019313 (ChemDiv C498-0579). For FOS experiments, 839 parasites were left in FOS only or supplemented with $5 \mu \mathrm{M}$ farnesol (Sigma F203), $5 \mu \mathrm{M}$ 840 geranylgeraniol (Sigma G3278), $5 \mu \mathrm{M}$ decaprenol (Isoprenoids polyprenol C50), $5 \mu \mathrm{M} \beta$-carotene 841 (Sigma C9750), $50 \mu \mathrm{M}$ DL-mevalonolactone (Cayman Chemicals 20348), or $200 \mu \mathrm{M} \mathrm{IPP}\left(\mathrm{NH}_{4}^{+}\right.$

842 salt, Isoprenoids IPP001). All parasites were cultured for 36 hours after synchronization and then

843 imaged by live-cell fluorescence microscopy to monitor apicoplast status. All concentrations 844 reflect the final concentration in culture medium.

846 Parasite synchronization: Parasites were synchronized to the ring stage either by treatment with

847 5\% D-sorbitol (Sigma S7900) or by first magnet-purifying schizonts and then incubating them 848 with uninfected erythrocytes for $5 \mathrm{hr}$ followed by treatment with sorbitol. Results from growth 849 assays and microscopy analyses using either of these synchronization methods were 850 indistinguishable within error, and 5\% sorbitol was used unless stated otherwise. 
852 Delayed Mevalonate-Rescue Assay: NF54 PfMev parasites were synchronized with 5\% (w/v)

853 D-sorbitol for 10 minutes at room temperature and returned to culture in $10 \mu \mathrm{M}$ fosmidomycin. 50

$854 \mu \mathrm{M}$ DL-mevalonate was added to cultures immediately or after 30,34 , or 38 hours post

855 synchronization. Parasitemia was measured by flow cytometry every 24 hours. After 60 hours

856 post-synchronization, parasites from each mevalonate time point were cloned out by limiting

857 dilution. Apicoplast status of all isolated clones was evaluated by live-cell $\mathrm{ACP}_{\mathrm{L}}-\mathrm{GFP}$

858 fluorescence. $\mathrm{ACP}_{\mathrm{L}}$-GFP signal was observed for the presence of distinct branching morphology

859 (apicoplast intact) or the presence of scattered punctate signals throughout the cytosol (apicoplast

860 disruption). A total of 9, 17, 18, and 5 clones from the $0,30,34$, and 38-hour rescue time-points,

861 respectively, were evaluated by microscopy (only 5 clones returned from the 38-hour rescue time

862 point). Apicoplast (SufB: Pf3D7_API04700) and nuclear (PPS: Pf3D7_0202700) genome PCR

863 (primers 4/5 and 1/2) and mevalonate-dependence growth assays were done on 2 clones from each

864 time point to confirm apicoplast status.

865

866 Parasite Culturing and Transfection: All experiments were performed using Plasmodium

867 falciparum Dd2, $\mathrm{ACP}_{\mathrm{L}}-\mathrm{GFP} \mathrm{D} 10^{28}$, or $\mathrm{ACP}_{\mathrm{L}}-\mathrm{GFP}$ NF54 $\mathrm{PfMev}^{29}$ parasite strains. Parasite

868 culturing was performed in Roswell Park Memorial Institute medium (RPMI-1640, Thermo Fisher

86923400021 ) supplemented with $2.5 \mathrm{~g} / \mathrm{L}$ Albumax I Lipid-Rich BSA (Thermo Fisher 11020039), 15

$870 \mathrm{mg} / \mathrm{L}$ hypoxanthine (Sigma H9636), $110 \mathrm{mg} / \mathrm{L}$ sodium pyruvate (Sigma P5280), $1.19 \mathrm{~g} / \mathrm{L}$ HEPES

871 (Sigma H4034), $2.52 \mathrm{~g} / \mathrm{L}$ sodium bicarbonate (Sigma S5761), 2 g/L glucose (Sigma G7021), and

$87210 \mathrm{mg} / \mathrm{L}$ gentamicin (Invitrogen Life Technologies 15750060). Cultures were generally

873 maintained at $2 \%$ hematocrit in human erythrocytes obtained from the University of Utah Hospital

874 blood bank, at $37{ }^{\circ} \mathrm{C}$, and at $5 \% \mathrm{O}_{2}, 5 \% \mathrm{CO}_{2}, 90 \% \mathrm{~N}_{2}$. Parasite-infected erythrocytes were 
875 transfected in 1X cytomix containing 50-100 $\mu \mathrm{g}$ midi-prep DNA by electroporation in $0.2 \mathrm{~cm}$

876 cuvettes using a Bio-Rad Gene Pulser Xcell system $(0.31 \mathrm{kV}, 925 \mu \mathrm{F})$. Transgenic parasites were

877 selected on the basis of plasmid resistance cassettes encoding human $\mathrm{DHFR}^{36}$, yeast $\mathrm{DHOD}^{79}$, or

878 blasticidin-S deaminase $(\mathrm{BSD})^{35}$ and cultured in $5 \mathrm{nM}$ WR99210, $2 \mu \mathrm{M}$ DSM1, or $6 \mu \mathrm{M}$

879 blasticidin-S, respectively. Gene-edited Dd2 parasites that contained PPS (PF3D7_0202700)

880 tagged with the aptamer/TetR-DOZI cassette ${ }^{57}$ were maintained in $0.5-1 \mu \mathrm{M}$ anhydrotetracycline

881 (Caymen Chemicals 10009542). Genetically modified parasites were genotyped by PCR and/or

882 Southern blot, as previously described. ${ }^{80}$ For western blot and IFA studies of PPS-GFP in

883 apicoplast-disrupted Dd2 parasites, transgenic parasites were cultured $>7$ days in 5 nM WR99210,

$8841 \mu \mathrm{M}$ doxycycline (Sigma D9891), and $200 \mu \mathrm{M}$ IPP to induce stable apicoplast loss prior to

885 parasite harvest.

886

887 Parasite Growth Assays: Parasite growth was monitored by diluting asynchronous or sorbitol-

888 synchronized parasites to $\sim 0.5 \%$ parasitemia and allowing culture expansion over several days

889 with daily media changes. Parasitemia was monitored daily by flow cytometry by diluting $10 \mu 1$

890 of each parasite culture well from each of two to three biological replicate samples into $200 \mu 1$ of

$891 \quad 1.0 \mu \mathrm{g} / \mathrm{ml}$ acridine orange (Invitrogen Life Technologies A3568) in phosphate buffered saline

892 (PBS) and analysis on a BD FACSCelesta system monitoring SSC-A, FSC-A, PE-A, FITC-A, and

893 PerCP-Cy5-5-A channels. Daily parasitemia measurements for asynchronous cultures were plotted

894 as function of time and fit to an exponential growth equation using GraphPad Prism 9.0. For EC 50

895 determinations, synchronous ring-stage parasites were diluted to $1 \%$ parasitemia and incubated

896 with variable drug concentrations for 48-72 hours without media changes. Parasitemia was

897 determined by flow cytometry in biological duplicate samples for each drug concentration, 
normalized to the parasitemia in the absence of drug, plotted as a function of the log of the drug concentration (in $\mathrm{nM}$ or $\mu \mathrm{M}$ ), and fit to a 4-parameter dose-response model using GraphPad Prism 9.0 .

901

Cloning and Episomal Expression of PPS: The gene encoding PPS (PF3D7_0202700) lacks

903 introns and was cloned by PCR from Dd2 parasite genomic DNA using primers designed for 904 insertion into the XhoI/AvrII sites of pTYEOE (yeast DHOD positive selection cassette) ${ }^{81}$ and 905 pTEOE (human DHFR positive selection cassette) ${ }^{55}$ vectors in frame with C-terminal RFP and 906 GFP tags, respectively. These vectors are designed to drive episomal protein expression using the 907 HSP86 promoter and for co-transfection with plasmid pHTH that contains the piggyBac 908 transposase ${ }^{82}$ for integration into the parasite genome. A single forward primer was used for PPS 909 cloning into both vectors (primer 1) while reverse primers were vector-specific (primers 2 and 3) 910 Cloning was completed using ligation-independent cloning (QuantaBio RepliQa HiFi Assembly 911 Mix). Cloning products were transformed into Top10 chemically competent cells, and bacterial 912 clones were selected for carbenicillin (Sigma C3416) resistance. Correct plasmid sequence in 913 isolated clonal bacteria was confirmed by both AscI/AatII (NEB) restriction digest and Sanger 914 sequencing (University of Utah DNA Sequencing Core). $100 \mu \mathrm{g}$ of either purified PPS-RFP915 TyEOE or PPS-GFP-TEOE in combination with $25 \mu \mathrm{g}$ of the pHTH transposase plasmid was 916 transfected into Dd2 parasites by electroporation, as described above. Transfected parasites were 917 allowed to expand in the absence of drug for 48 hours before selection with either $2 \mu \mathrm{M}$ DSM1 or 9185 nM WR99210 for PPS-RFP-TyEOE or PPS-GFP-TEOE respectively. Stable, drug-resistant 919 parasites returned from transfection in 3-6 weeks. 


\section{PPS Gene-Editing to Enable Ligand-Dependent Regulation of Protein Expression:}

922 Crispr/Cas9-stimulated repair by double-crossover homologous recombination was used to tag the

923 PPS gene (PF3D7_0202700) to encode a C-terminal hemagglutinin (HA)-FLAG epitope tag and

924 the $3^{\prime} 10 \mathrm{X}$ aptamer/TetR-DOZI system $^{57}$ to enable regulated PPS expression using

925 anhydrotetracycline. Guide RNA sequences corresponding to TGATATAAAACAAAGTAGCG,

926 CGTGCTAGTTCTATTTTTGC, and GATGATTCAAATAAAAGAAG (primers 6-11) were

927 cloned into a modified version of the previously published pAIO vector, ${ }^{83}$ in which the BtgZI site

928 was replaced with a unique HindIII site to facilitate cloning (primers 12 and 13). To tag the PPS

929 gene, a donor pMG75 ${ }^{57}$ repair plasmid was prepared by PCR-amplifying 635 bp of the 3' coding

930 sequence and $679 \mathrm{bp}$ of the 3' untranslated region (UTR) as homology flanks to the PPS gene,

931 fusing these fragments together by PCR with an AflII site in between (679 bp 3' UTR-AflII-635

932 bp 3' coding sequence), and inserting this fused fragment into the AscI and AatII sites of the

933 pMG75 vector (primers 14-17). A shield mutation was introduced to the 3 ' end of the coding-

934 sequence homology flank corresponding to the gRNA sequence

935 TGATATAAAACAAAGTAGCG. This mutation (introduced using primer 18) ablated the

936 CRISPR PAM sequence AGG that immediately following the gRNA sequence above by mutating

937 it to AAG, resulting in a silent mutation of the Glu523 codon from GAG to GAA. Sanger

938 sequencing confirmed the correct sequence of the homology flanks inserted into the pMG75

939 vector. PCR analysis of the final pMG75 vector using primers 39-40 revealed that only 9 copies

940 of the aptamer sequence were retained. Before transfection, the pMG74 vector was linearized by

941 AflII digestion performed overnight at $37^{\circ} \mathrm{C}$, followed by deactivation with Antarctic Phosphatase

942 (NEB M0289S). 
944 linearized pMG75 donor plasmid, as described above. Parasites were selected on the basis of the

945 BSD resistance cassette encoded by the pMG75 plasmid and returned from transfection after 4-6

946 weeks. Gene-edited Dd2 parasites resulting from transfection with pAIO Cas9/gRNA-4 (produced

947 with primers 10/11) contained PPS (PF3D7_0202700) tagged with the aptamer/TetR-DOZI

948 cassette $^{57}$ and were maintained in 0.5-1 $\mu \mathrm{M}$ anhydrotetracycline (Caymen Chemicals 10009542).

949 Genetically modified parasites were genotyped by Southern blot, as previously described. ${ }^{80}$

950 Briefly, genomic DNA from the polyclonal parasites that returned from transfection was digested 951 with BamHI and SpeI (New England Biolabs) and transferred to membrane (Nytran SuPerCharge)

952 using the TurboBlotter system (VWR 89026-838). A DNA probe consisting of the 5' $750 \mathrm{bp}$ of 953 the PPS gene was produced by PCR (primers 16/17). Probe labeling, hybridization, and 954 visualization was performed using the AlkPhos Direct Labeling and Detection System (VWR 955 95038-288) and CDP-Star reagent (VWR 95038-292). The Southern blot confirmed complete 956 integration into the PPS locus without evidence for unmodified parasites, and the polyclonal 957 parasites were used for all subsequent experiments.

Analysis of PPS transcript levels: Cultures of PPS-aptamer/TetR-DOZI parasites were

960 synchronized in 5\% D-sorbitol and grown for 72 or 120 hours \pm aTc prior to harvest. 4-ml cultures

961 at approximately $10 \%$ were harvested by centrifugation $\left(2000 \mathrm{rpm}\right.$ for $3 \mathrm{~min}$.) and stored at $-20{ }^{\circ} \mathrm{C}$

962 until use. Total RNA was isolated from frozen parasite-infected blood pellets using a modified

963 Trizol (Invitrogen) extraction protocol. $5 \mathrm{~mL}$ Trizol (Invitrogen) was added to thawed pellets on

964 ice, pipetted 20-30 times to resuspend, and pulse-vortexed 20 times for $15 \mathrm{~s}$. $2 \mathrm{~mL}$ chloroform was

965 added to each sample and vortexed, incubated on ice for 5 minutes, then spun for 10 minutes at $4 \mathrm{C}$ 
at 5000rpm without brake. The top, aqueous layer (approximately $3 \mathrm{~mL}$ ) was transferred to a new tube. $5 \mathrm{~mL}$ of isopropanol was added to each sample, gently mixed, and incubated at $-80{ }^{\circ} \mathrm{C}$ for 20 minutes or $-20{ }^{\circ} \mathrm{C}$ overnight. Samples were spun at $5000 \mathrm{rpm}$ for $30 \mathrm{~min}$, washed with freshly-

969 made solution of $70 \%$ ethanol, then spun again for $10 \mathrm{~min}$. Ethanol was removed and pellets were

970 dried 30 min on ice. RNA pellets were resuspended in RNAse-free water, quantitated, and used

971 immediately or stored at $-80{ }^{\circ} \mathrm{C} .1 \mu \mathrm{g}$ of RNA was DNAse-treated and reverse-transcribed using

972 Superscript IV kit (Invitrogen) with the addition of gene-specific reverse primers 31-38.

973 Subsequent cDNA was analyzed in duplicate through qPCR reactions with SYBR Green

974 fluorescent probe (Invitrogen) in a Roche Lightcycler. Cp values for PPS (primers 35-36) were

975 normalized to the average of 2 nuclear-encoded control genes (I5P, PF3D7_0802500; ADSL,

976 PF3D7_0206700; primers 31-34), then used to calculate relative +aTC/-aTC RNA abundance for

977 each of two biological replicates. Significance of the observed difference was evaluated by two-

978 tailed unpaired t-test using GraphPad Prism 9.0.

979

980 Synchronous Growth Assays of PPS Knockdown Parasites: Dd2 parasites tagged at the

981 genomic PPS locus with the aptamer/TetR-DOZI system were synchronized by 5\% D-sorbitol to

982 ring-stage parasites and allowed to expand $\pm \mathrm{aTc}$ in two or three biological replicate samples.

983 Parasitemia values were measured daily by flow cytometry and plotted as the average \pm SD of

984 replicate samples. For growth-rescue experiments, synchronous parasites were allowed to expand

$985 \pm \mathrm{aTc}$, and -aTc plus $200 \mu \mathrm{M}$ IPP, $5 \mu \mathrm{M}$ farnesol (FOH), $5 \mu \mathrm{M}$ geranylgeraniol (GGOH), or $5 \mu \mathrm{M}$

986 decaprenol $\left(\mathrm{C}_{50}-\mathrm{OH}\right)$. For growth-rescue experiments involving fosmidomycin (FOS), PPS KD

987 parasites were synchronized to rings with 5\% D-sorbitol and grown for 4 days (96 hours) \pm aTc.

988 After 96 hours, all culture wells were synchronized again with 5\% D-sorbitol and supplemented 
with $10 \mu \mathrm{M}$ FOS and $200 \mu \mathrm{M}$ IPP, $5 \mu \mathrm{M} \mathrm{FOH}, 5 \mu \mathrm{M}$ GGOH, or $5 \mu \mathrm{M}$ decaprenol. Parasites were

cultured for another 38 hours before harvest at 134 total hours post-initial synchronization for IFA

991 analysis of apicoplast morphology. Parasites grown \pm aTc with $10 \mu \mathrm{M}$ FOS and $200 \mu \mathrm{M}$ IPP were

992 allowed to expand for an additional 48 hours and harvested at 182 hours post-initial

993 synchronization for analysis by IFA and qPCR for apicoplast morphology and apicoplast:nuclear

994 genome levels, respectively.

995

996 qPCR Analysis of Apicoplast:Nuclear genomic DNA levels: Genomic DNA was extracted from

997 parasite samples grown \pm aTc with $10 \mu \mathrm{M}$ FOS and $200 \mu \mathrm{M}$ IPP and harvested at 182 hours post-

998 initial synchronization. DNA extraction was performed using the QIAmp DNA Blood Mini Kit

999 (Qiagen 51104). Primers for qPCR were designed to amplify a 120-140 bp region of an apicoplast

1000 gene (TufA, PF3D7_API02900, primers 35-36) and each of two nuclear genes (I5P,

1001 PF3D7_0802500; ADSL, PF3D7_0206700; primers 31-34). Approximately 100 ng of DNA was

1002 amplified in each of two biological replicates with PowerUp SYBR Green Master Mix

1003 (ThermoFisher A25741) in a 96-well plate with $20 \mu 1$ reaction volume on a Quantstudio3 Real

1004 Time PCR system. Specificity of primer amplification was confirmed for every sample by

1005 identifying only one melting temperature for the product of each qPCR reaction. Abundance of

1006 apicoplast relative to nuclear DNA was determined by comparative $\mathrm{C}_{\mathrm{t}}$ analysis, ${ }^{84}$ with

1007 amplification of TufA (apicoplast) and I5P (nuclear) and calculation of $2^{\Delta \mathrm{Ct}}$, where $\Delta \mathrm{Ct}=\mathrm{Ct}_{\mathrm{TufA}}-$

$1008 \mathrm{Ct}_{15 \mathrm{P}}$. As a positive control, abundance of a second nuclear gene (ADSL) relative to I5P was

1009 calculated similarly. The $2^{\Delta \mathrm{Ct}}$ value for TufA or ADSL was normalized to $+\mathrm{aTc}$ for each gene to

1010 determine a normalized target gene:control gene DNA abundance. Error bars represent the 
1011 standard deviation between replicates, and $\mathrm{P}$ values were determined by two-tailed unpaired t-test 1012 in GraphPad Prism 9.0.

1013

1014 MiaA Gene Disruption: The gene encoding MiaA (PF3D7_1207600) was disrupted in the NF54 1015 PfMev line using CRISPR/Cas9 and gene deletion by double-crossover homologous 1016 recombination, similar to the recently described disruption of the DXPR gene (PF3D7_1467300). ${ }^{29}$ 1017 Homology arm regions (411 bp for the 5' arm and $540 \mathrm{bp}$ for the 3' arm) were PCR-amplified 1018 from genomic DNA with primers 19-22 and cloned into the vector $\mathrm{pRS}^{29}$ using ligation1019 independent cloning (In-Fusion, Clontech). A guide RNA with sequence 1020 AATAACGATATTAAATGTAA was cloned into a modified pAIO vector called pCasG ${ }^{85}$ using 1021 primers 23 and $24.75 \mu \mathrm{g}$ of pRS-miaA-KO plasmid was combined with $75 \mu \mathrm{g}$ of the pCasG guide 1022 RNA plasmid and transfected into NF54 PfMev parasites. Transfected parasites were allowed to 1023 expand for 48 hours in $50 \mu \mathrm{M}$ mevalonate before selection with $5 \mathrm{nM}$ WR99210 and $50 \mu \mathrm{M}$ 1024 mevalonate. Parasites returning from positive selection were genotyped by PCR using primers 25102530 . Asynchronous growth of $\Delta$ MiaA PfMev parasites \pm Mev compared to parental PfMev parasites 1026 was performed on biological duplicate samples. Average parasitemia values \pm SD were plotted 1027 versus time and fit to an exponential growth equation in GraphPad Prism 9.0. Apicoplast (SufB: 1028 Pf3D7_API04700) and nuclear (LDH: PF3D7_1324900) genome PCR was performed to confirm 1029 apicoplast status in parental PfMev and $\Delta$ MiaA parasites, as previously reported. ${ }^{29}$

1031 Western Blots: Samples of episomal PPS-GFP Dd2 or endogenously HA-FLAG-tagged PPS Dd2 1032 parasites were harvested by centrifugation and treated with $0.05 \%$ saponin (Sigma 84510 ) in PBS 1033 for $5 \mathrm{~min}$ at room temperature and spun down by centrifuge at 5,000 rpm for 30 minutes at $4^{\circ} \mathrm{C}$. 
$10342 \%$ SDS was added to saponin pellets and resuspended by sonication. Parasites were incubated in $10352 \%$ SDS overnight at $4{ }^{\circ}$ C. $5 x$ Sample buffer containing beta-mercaptoethanol (BME) was added

1036 to parasite samples before heating at $95^{\circ} \mathrm{C}$ for 5 minutes and centrifuging at 13,000 rpm for 5

1037 minutes. Samples were fractionated by SDS-polyacrylamide gel electrophoresis (PAGE) using

$103810 \%$ acrylamide gels run at $120 \mathrm{~V}$ in the BIO-RAD mini-PROTEAN electrophoresis system.

1039 Fractionated proteins were transferred from polyacrylamide gel to a nitrocellulose membrane at $1040100 \mathrm{~V}$ for one hour using the BIO-RAD wet transfer system. Membranes were blocked in $1 \%$ 1041 casein/PBS for one hour at room temperature and then probed with primary antibody overnight at $10424^{\circ} \mathrm{C}$ and secondary antibody at room temperature for 1 hour. Episomal PPS-RFP parasite samples 1043 were probed with 1:1000 mouse anti-RFP (Invitrogen Life Technologies MA5-15257) and 1044 1:10,000 donkey anti-mouse DyLight800 (Invitrogen Life Technologies SA5-10172). Endogenous 1045 HA-FLAG-tagged PPS parasite samples were probed with Roche rat anti-HA monoclonal 3F10 1046 primary (Sigma 11867423001) and donkey-anti-rat DyLight800 (Invitrogen Life Technologies 1047 SA5-10032) secondary antibodies.

1049 Sequence Similarity Analysis and Structural Homology Modeling: Sequence similarity 1050 searches for P. falciparum homologs to chicken FPPS (Uniprot P08836) were performed by 1051 BLASTP analysis as implemented at the Plasmodium Genomics Resource webpage 1052 (www.plasmodb.org). Sequence similarity searches using the PPS (PF3D7_0202700) protein 1053 sequence as query were carried out using NCBI BLAST ${ }^{51}$ (excluding organisms in the phylum 1054 Apicomplexa to which $P$. falciparum belongs) and MPI HHpred ${ }^{52}$. A homology model of PPS was 1055 generated by the MPI HHpred software using the X-ray crystallographic structural model of $E$. 
1056 coli OPPS (PDB 3WJK), which was one of the top 10 homology hits by HHpred analysis, as

1057 template. Structural models were visualized using PyMol (Schrödinger).

1058

$1059 \beta$-Carotene Extraction and Analysis by Mass Spectrometry: For determination of beta-

1060 carotene levels in parasite-infected versus uninfected erythrocytes, $35 \mathrm{ml}$ of $4 \%$ hematocrit $P$.

1061 falciparum culture infected at 13-15\% parasitemia with the Dd2 PPS aptamer/TetR-DOZI

1062 knockdown parasites were collected after 6 days of growth in the presence or absence of $1 \mu \mathrm{M}$

1063 aTc. Uninfected erythrocyte samples were prepared by collecting $20 \mathrm{ml}$ of 4\% hematocrit

1064 uninfected culture incubated for 6 days in RPMI media that lacked or contained $2.5 \mathrm{~g} / \mathrm{L}$ AlbuMAX.

1065 Samples of infected or uninfected erythrocytes were harvested by centrifugation, lysed by $0.05 \%$

1066 saponin, and pelleted by centrifugation. Saponin pellets were washed in PBS and then extracted

1067 three times in $1 \mathrm{ml}$ of chilled acetone (pellet was briefly sonicated after addition of the first acetone

1068 volume). The supernatant of each extraction was pooled and dried down by vacuum concentration

1069 (Speed Vac). Three biological replicates of each sample were prepared. For analysis of AlbuMAX,

$107085 \mathrm{mg}$ of dry AlbuMAX (equivalent to the AlbuMAX content in $35 \mathrm{ml}$ of complete culture media)

1071 was extracted in 3 volumes of cold acetone, and supernatants were combined and dried as above.

1072 For analysis of $\beta$-carotene synthesis, NF54 PfMev parasites were cultured and expanded

1073 over three intraerythrocytic cycles in media containing $50 \mu \mathrm{M} 2-{ }^{13} \mathrm{C}$-mevalonate and $10 \mu \mathrm{M}$

1074 fosmidomycin. This strategy was chosen to inhibit MEP pathway activity, ensure full ${ }^{13} \mathrm{C}$-labeling

1075 of the endogenous IPP and DMAPP precursor pool within parasites produced by the cytoplasmic

1076 bypass enzymes, and result in a distinguishable 8 Da mass increase for any $\beta$-carotene derived

1077 from de novo synthesis. Final parasite samples contained $70 \mathrm{ml}$ of culture at $15 \%$ parasitemia and 
1078 were collected by centrifugation prior to $0.05 \%$ saponin lysis, centrifugation, and washing the 1079 pellet in PBS. The final pellet was extracted in acetone and dried, as described above.

1081 biosynthesized $\beta$-carotene, we turned to studies of $E$. coli bacteria engineered to biosynthesize $\beta$ 1082 carotene ${ }^{64}$ and grown in minimal M9 medium with uniformly labeled ${ }^{13} \mathrm{C}$-glucose as the sole 1083 carbon source. Growth of bacteria in these conditions was expected to lead to a 40-Da mass 1084 increase in detected $\beta$-carotene. 5-ml cultures of pAC-BETAipi E. coli or untransformed Top 10 1085 E. coli were allowed to expand over two days at $30^{\circ} \mathrm{C}$ in the dark. Bacterial cultures were harvested 1086 by centrifugation at 5,000 rpm for $10 \mathrm{~min}$., and bacterial pellets were extracted three times in cold 1087 acetone and dried, as described above. purchased from VWR. Samples were resuspended in $50 \mu 1 \mathrm{MeOH} / \mathrm{CHCl}_{3}(2 \mathrm{mM} \mathrm{LiI})$, and a sample volume of $10 \mu \mathrm{l}$ was injected onto a Phenomenex Luna 150 x $2.1 \mathrm{~mm}$ reverse-phase C8 column 1091 maintained at $30^{\circ} \mathrm{C}$ and connected to an Agilent HiP 1290 Sampler, Agilent 1290 Infinity pump, 1092 and Agilent 6545 Accurate Mass Q-TOF dual AJS-ESI mass spectrometer. The instrument was 1093 operated in positive ion mode, and the source gas temperature was $275^{\circ} \mathrm{C}$ with a drying gas flow 1094 of $12 \mathrm{~L} / \mathrm{min}$, nebulizer pressure of $35 \mathrm{psig}$, sheath gas temp of $325^{\circ} \mathrm{C}$ and sheath gas flow of 12 $1095 \mathrm{~L} / \mathrm{min}$. VCap voltage was set at $3500 \mathrm{~V}$, nozzle voltage $250 \mathrm{~V}$, fragmentor at $90 \mathrm{~V}$, skimmer at 65 $1096 \mathrm{~V}$, octopole RF peak at $750 \mathrm{~V}$ and a scan range $\mathrm{m} / z 40$ - 900. The mobile solvent phase $\mathrm{A}$ was $\mathrm{H}_{2} \mathrm{O}$ 1097 with $0.1 \%$ formic acid, and mobile phase B was MeOH:ACN:IPA $(2: 2: 1 \mathrm{v} / \mathrm{v})$ with $0.1 \%$ formic 1098 acid. The chromatography gradient started at 80\% mobile phase B then increased to $100 \%$ B over 10996 min where it was held until $9.9 \mathrm{~min}$ and then returned to the initial conditions and equilibrated 1100 for $5 \mathrm{~min}$. The column flow rate was $0.5 \mathrm{~mL} / \mathrm{min}$. 
1102 Workstation and analyzed using the software packages MH Qual and MH Quant (Agilent

1103 Technologies, Inc.). Unlabeled, $2-{ }^{13} \mathrm{C}$-mevalonate-labeled, and uniform ${ }^{13} \mathrm{C}$-glucose-labeled $\beta$ -

1104 carotene were analyzed using the molecular ions of $\mathrm{m} / \mathrm{z} 536.4377, \mathrm{~m} / \mathrm{z} 544.4645$, and $\mathrm{m} / \mathrm{z}$

1105576.5718 , respectively. Fragmentation profiling of unlabeled $\beta$-carotene by MS/MS confirmed the

1106 expected product ions at $\mathrm{m} / \mathrm{z} 444$ and $\mathrm{m} / \mathrm{z} 119$, as previously reported. ${ }^{86}$ For quantitation of

1107 unlabeled $\beta$-carotene levels in experimental samples and determination of a limit of detection

1108 (LOD), a calibration curve was constructed using serial dilutions of commercial ß-carotene (Sigma

1109 C9750). The concentration LOD for a 10- $\mu$ l sample of unlabeled $\beta$-carotene in this assay was 2.6

$1110 \mathrm{ng} / \mathrm{mL}$. Integrated peak areas for unlabeled $\beta$-carotene in experimental samples were converted to

1111 concentration values in the $10-\mu \mathrm{L}$ sample using this calibration curve.

\begin{tabular}{|c|c|c|}
\hline \multicolumn{3}{|c|}{ Primers used in this study } \\
\hline No. & Name & Sequence (5' to 3') \\
\hline 1 & PPS-EOE-F & ACACGATTTTTTCTCGAGATGGTTCACCTAAGTAAAAGAAATAATATTAAAAGCTTTTTA \\
\hline 2 & PPS-GFP-TEOE-R & TGCTGCACCTGGCCTAGGTTTGACGTTTCTTGATAACACGTTTAAGATTAAATTAATTAA \\
\hline 3 & PPS-RFP-TyEOE-R & TCAATTAAGTTTCCTAGGTTTGACGTTTCTTGATAACACGTTTAAGATTAAATTAATTAA \\
\hline 4 & SufB-F & ACGATTTTTTCTCGAGATGATAAAATTAAAAAATTTTTTAAATATTTATAATTTAAATTA \\
\hline 5 & SufB-R & TAGACACCATCCTAGGATTAAATATATCTTTAATTTTTAATGAAAATAATATAGGTATCT \\
\hline 6 & PPS-Cas9gRNA1-F & TAAGTATATAATATTCGTGCTAGTTCTATTTTTGCGTTTTAGAGCTAGAA \\
\hline 7 & PPS-Cas9gRNA1-R & TTCTAGCTCTAAAACGCAAAAATAGAACTAGCACGAATATTATATACTTA \\
\hline 8 & PPS-Cas9gRNA2-F & TAAGTATATAATATTGATGATTCAAATAAAAGAAGGTTTTAGAGCTAGAA \\
\hline 9 & PPS-Cas9gRNA2-R & TTCTAGCTCTAAAACCTTCTTTTATTTGAATCATCAATATTATATACTTA \\
\hline 10 & PPS-Cas9gRNA4-F & TAAGTATATAATATT TGATATAAAACAAAGTAGCG GTTTTAGAGCTAGAA \\
\hline 11 & PPS-Cas9gRNA4-R & TTCTAGCTCTAAAACCGCTACTTTGTTTTATATCAAATATTATATACTTA \\
\hline 12 & Cas9HindIII-F & AATATTAAGCTTGTTTTAGAGCTAGAAATAGCAAGTTAAAATAAGGCTAGTCCGTTATCA \\
\hline 13 & Cas9HindIII-R & $\begin{array}{l}\text { TAAAACAAGCTTAATATTATATACTTAATATGAAATATGTGCATATAGGAAAAATTATGCA } \\
\text { TTTTGGTTACTCTAATATTATATATATAT }\end{array}$ \\
\hline 14 & PPS_3'UTR_HF-F & $\begin{array}{l}\text { GGCCCCTTTCCGGGCGCGCCCAATAACATATACAATATCAAACATATATATTATAATATTA } \\
\text { TTAAACATCTTCAATATTGTATTATTTAA }\end{array}$ \\
\hline 15 & PPS_3'UTR_HF-R & $\begin{array}{l}\text { TAATGCTATGACACCTCTTCTTTTATTTGACTTAAGTATGTTTGATACATGTAGATTTCTTA } \\
\text { AAGAATGAAGCTTA }\end{array}$ \\
\hline 16 & PPS 3'CR_HF-F & TTTAAGAAATCTACATGTATCAAACATACTTAAGTCAAATAAAAGAAGAGGTGTCATAGC \\
\hline 17 & PPS_3'CR_HF-R & GTCATAAGGATAGACGTCATCATTTGACGTTTCTTGATAACACG \\
\hline 18 & PPS_3'CR_HF-SM-R & $\begin{array}{l}\text { CATAAGGATAGACGTCTTTGACGTTTCTTGATAACACGTTTAAGATTAAATTAATTAATGC } \\
\text { TTCGCTACTTTGTTTTATATCATCATGTT }\end{array}$ \\
\hline 19 & miaA.HA1.F & GCCACGAGCGGCCGTAAATTAAAGACAACGGGCTGTCAAC \\
\hline 20 & miaA.HA1.R & AAGCGCAGCGGCCGGGAATTTCCATCTCTAAAAAAGTTCA \\
\hline 21 & miaA.HA2.F & CGACAGACGCCGGTGAAAGAAATGATGATATGGTAGAATT \\
\hline 22 & miaA.HA2.R & GGCCACCAGCCGGCGATATCCATCTTCTTTGTTTCTTGGC \\
\hline 23 & miaA.gRNA.F & TAAGTATATAATATTAATAACGATATTAAATGTAAGTTTTAGAGCTAGAA \\
\hline 24 & miaA.gRNA.R & TTCTAGCTCTAAAACTTACATTTAATATCGTTATTAATATTATATACTTA \\
\hline
\end{tabular}




\begin{tabular}{|l|l|l|}
\hline 25 & miaA.5.F & GTTGAATAAATAAATGCCTCTCTATATATTGTTAACAT \\
\hline 26 & miaA.5.WT.R & CTTCGACTTTAGCAATACCTACATTG \\
\hline 27 & miaA.3.F & GGGAATCAGTAATTGATATAAGAAAAGAAG \\
\hline 28 & miaA.3.WT.R & AAACTTCAAGACAATGCCTATAGC \\
\hline 29 & pRS.F & CATATTTATTAAATCTAGAATTCGACAGACGCCGG \\
\hline 30 & pRS.R & TACAAAATGCTTAAGCGCAGCGGCC \\
\hline 31 & I5P-qPCR-F & GACATAAGTTAGTAGGTCG \\
\hline 32 & I5P-qPCR-R & TTCTGACTCCACATCATTTG \\
\hline 33 & ADSL-qPCR-F & GGAAATCCATAGACAAACAATG \\
\hline 34 & ADSL-qPCR-R & TCCTGTGAGAAGTGCTCCAC \\
\hline 35 & TufA-qPCR-F & AAGATGTATTTTCTATAACAGGTAGAGGTA \\
\hline 36 & TufA-qPCR-R & AACTGTTGTTAAATTAGGAGATGATTTTTC \\
\hline 37 & PPS-qPCR-F & ATCAGGGGATTATCTCTTAGCAC \\
\hline 38 & PPS-qPCR-R & AACTTTCGACAACATAAGAGAAACT \\
\hline 39 & 3'APT-F & CTTATGACGTACCTGATTATGCAC \\
\hline 40 & $10 x$ APT-R & GTAGACCCCATTGTGAGTACATAAATATATTATATAAACTAGACTAGG \\
\hline
\end{tabular}

1115 members of the Sigala lab for helpful discussions. Research reported in this publication was 1116 supported by Department of Defense PRMRP Discovery Award W81XWH1810060 (to PAS), 1117 National Institutes of Health grants R35GM133764 (to PAS) and AI125534 (to STP), a Burroughs 1118 Wellcome Fund Career Award at the Scientific Interface (to PAS), the Johns Hopkins Malaria 1119 Research Institute (STP), and the Bloomberg Family Foundation (STP). PAS is a Pew Scholar in 1120 the Biomedical Sciences, supported by The Pew Charitable Trusts. MO and KR were supported in 1121 part by NIH training grants T32DK007115 and T32AI007417, respectively. Metabolomics 1122 analyses were supported in part by NIH grant U54DK110858. DNA synthesis and sequencing, 1123 epifluorescence microscopy, mass spectrometry metabolomics, generation of CRISPR/Cas9 1124 reagents, and flow cytometry were performed using core facilities at the University of Utah. Mass 1125 spectrometry equipment was obtained through NCRR Shared Instrumentation Grants 1126 1S10OD016232-01, 1S10OD018210-01A1 and 1S10OD021505-01. 


\section{REFERENCES}

1129

1130

1131

1132

1133

1134

1135

1136

1137

1138

1139

1140

1141

1142

1143

1144

1145

1146

1147

1148

1149

1150

1151

1152

1153

1154

1155

1156

1157

1158

1159

1160

1161

1162

1163

1164

1165

1166

1167

1168

1169

1170

1171

1172

[1] Ralph, S. A., van Dooren, G. G., Waller, R. F., Crawford, M. J., Fraunholz, M. J., Foth, B. J., Tonkin, C. J., Roos, D. S., and McFadden, G. I. (2004) Metabolic maps and functions of the Plasmodium falciparum apicoplast, Nat. Rev. Microbiol. 2, 203-216.

[2] Ke, H., Sigala, P. A., Miura, K., Morrisey, J. M., Mather, M. W., Crowley, J. R., Henderson, J. P., Goldberg, D. E., Long, C. A., and Vaidya, A. B. (2014) The heme biosynthesis pathway is essential for Plasmodium falciparum development in mosquito stage but not in blood stages, J. Biol. Chem. 289, 34827-34837. DOI: https://doi.org/10.1074/jbc.M114.615831

[3] Goldberg, D. E., and Sigala, P. A. (2017) Plasmodium heme biosynthesis: To be or not to be essential?, PLoS Pathog. 13, e1006511. DOI: https://doi.org/10.1371/journal.ppat.1006511

[4] Yu, M., Kumar, T. R. S., Nkrumah, L. J., Coppi, A., Retzlaff, S., Li, C. D., Kelly, B. J., Moura, P. A., Lakshmanan, V., Freundlich, J. S., Valderramos, J. C., Vilcheze, C., Siedner, M., Tsai, J. H. C., Falkard, B., Sidhu, A. B. S., Purcell, L. A., Gratraud, P., Kremer, L., Waters, A. P., Schiehser, G., Jacobus, D. P., Janse, C. J., Ager, A., Jacobs, W. R., Sacchettini, J. C., Heussler, V., Sinni, P., and Fidock, D. A. (2008) The Fatty Acid Biosynthesis Enzyme Fabl Plays a Key Role in the Development of Liver-Stage Malarial Parasites, Cell Host Microbe 4, 567-578. DOI: https://doi.org/10.1016/J.Chom.2008.11.001

[5] Shears, M. J., Botte, C. Y., and McFadden, G. I. (2015) Fatty acid metabolism in the Plasmodium apicoplast: Drugs, doubts and knockouts, Mol. Biochem. Parasitol. 199, 3450. DOI: https://doi.org/10.1016/j.molbiopara.2015.03.004

[6] Dahl, E. L., and Rosenthal, P. J. (2007) Multiple antibiotics exert delayed effects against the Plasmodium falciparum apicoplast, Antimicrob. Agents Chemother. 51, 3485-3490. DOI: https://doi.org/10.1128/AAC.00527-07

[7] Guggisberg, A. M., Amthor, R. E., and Odom, A. R. (2014) Isoprenoid biosynthesis in Plasmodium falciparum, Eukaryot. Cell 13, 1348-1359. DOI: https://doi.org/10.1128/EC.00160-14

[8] Jomaa, H., Wiesner, J., Sanderbrand, S., Altincicek, B., Weidemeyer, C., Hintz, M., Turbachova, I., Eberl, M., Zeidler, J., Lichtenthaler, H. K., Soldati, D., and Beck, E. (1999) Inhibitors of the nonmevalonate pathway of isoprenoid biosynthesis as antimalarial drugs, Science 285, 1573-1576. DOI:

https://doi.org/10.1126/science.285.5433.1573

[9] van Dooren, G. G., Stimmler, L. M., and McFadden, G. I. (2006) Metabolic maps and functions of the Plasmodium mitochondrion, FEMS Microbiol. Rev. 30, 596-630. DOI: https://doi.org/10.1111/j.1574-6976.2006.00027.x

[10] Simao-Gurge, R. M., Wunderlich, G., Cricco, J. A., Cubillos, E. F. G., Domenech-Carbo, A., Cebrian-Torrejon, G., Almeida, F. G., Cirulli, B. A., and Katzin, A. M. (2019) Biosynthesis of heme $\mathrm{O}$ in intraerythrocytic stages of Plasmodium falciparum and potential inhibitors of this pathway, Sci. Rep. 9, 19261. DOI: https://doi.org/10.1038/s41598-019-55506-y

[11] Yeh, E., and DeRisi, J. L. (2011) Chemical rescue of malaria parasites lacking an apicoplast defines organelle function in blood-stage Plasmodium falciparum, PLoS Biol. 9, e1001138. DOI: https://doi.org/10.1371/journal.pbio.1001138 
1173

1174

1175

1176

1177

1178

1179

1180

1181

1182

1183

1184

1185

1186

1187

1188

1189

1190

1191

1192

1193

1194

1195

1196

1197

1198

1199

1200

1201

1202

1203

1204

1205

1206

1207

1208

1209

1210

1211

1212

1213

1214

1215

1216

1217

1218

[12] Uddin, T., McFadden, G. I., and Goodman, C. D. (2018) Validation of Putative ApicoplastTargeting Drugs Using a Chemical Supplementation Assay in Cultured Human Malaria Parasites, Antimicrob. Agents Chemother. 62. DOI: https://doi.org/10.1128/AAC.0116117

[13] Ramya, T. N., Mishra, S., Karmodiya, K., Surolia, N., and Surolia, A. (2007) Inhibitors of nonhousekeeping functions of the apicoplast defy delayed death in Plasmodium falciparum, Antimicrob. Agents Chemother. 51, 307-316. DOI: https://doi.org/10.1128/AAC.00808-06

[14] Kennedy, K., Crisafulli, E. M., and Ralph, S. A. (2019) Delayed Death by Plastid Inhibition in Apicomplexan Parasites, Trends Parasitol. 35, 747-759. DOI: https://doi.org/10.1016/j.pt.2019.07.010

[15] Kennedy, K., Cobbold, S. A., Hanssen, E., Birnbaum, J., Spillman, N. J., McHugh, E., Brown, H., Tilley, L., Spielmann, T., McConville, M. J., and Ralph, S. A. (2019) Delayed death in the malaria parasite Plasmodium falciparum is caused by disruption of prenylation-dependent intracellular trafficking, PLoS Biol. 17, e3000376. DOI: https://doi.org/10.1371/journal.pbio.3000376

[16] Amberg-Johnson, K., Hari, S. B., Ganesan, S. M., Lorenzi, H. A., Sauer, R. T., Niles, J. C., and Yeh, E. (2017) Small molecule inhibition of apicomplexan FtsH1 disrupts plastid biogenesis in human pathogens, Elife 6. DOI: https://doi.org/10.7554/eLife.29865

[17] Boucher, M. J., and Yeh, E. (2019) Disruption of Apicoplast Biogenesis by Chemical Stabilization of an Imported Protein Evades the Delayed-Death Phenotype in Malaria Parasites, mSphere 4. DOI: https://doi.org/10.1128/mSphere.00710-18

[18] Okada, M., Guo, P., Nalder, S. A., and Sigala, P. A. (2020) Doxycycline has distinct apicoplast-specific mechanisms of antimalarial activity, Elife 9. DOI: https://doi.org/10.7554/eLife.60246

[19] Gisselberg, J. E., Herrera, Z., Orchard, L. M., Llinas, M., and Yeh, E. (2018) Specific Inhibition of the Bifunctional Farnesyl/Geranylgeranyl Diphosphate Synthase in Malaria Parasites via a New Small-Molecule Binding Site, Cell Chem. Biol. 25, 185-193 e185. DOI: https://doi.org/10.1016/j.chembiol.2017.11.010

[20] Imlay, L., and Odom, A. R. (2014) Isoprenoid metabolism in apicomplexan parasites, Curr. Clin. Microbiol. Rep. 1, 37-50. DOI: https://doi.org/10.1007/s40588-014-0006-7

[21] Gisselberg, J. E., Dellibovi-Ragheb, T. A., Matthews, K. A., Bosch, G., and Prigge, S. T. (2013) The suf iron-sulfur cluster synthesis pathway is required for apicoplast maintenance in malaria parasites, PLoS Pathog. 9, e1003655. DOI: https://doi.org/10.1371/journal.ppat.1003655

[22] Gabriel, H. B., de Azevedo, M. F., Palmisano, G., Wunderlich, G., Kimura, E. A., Katzin, A. M., and Alves, J. M. (2015) Single-target high-throughput transcription analyses reveal high levels of alternative splicing present in the FPPS/GGPPS from Plasmodium falciparum, Sci. Rep. 5, 18429. DOI: https://doi.org/10.1038/srep18429

[23] No, J. H., de Macedo Dossin, F., Zhang, Y., Liu, Y. L., Zhu, W., Feng, X., Yoo, J. A., Lee, E., Wang, K., Hui, R., Freitas-Junior, L. H., and Oldfield, E. (2012) Lipophilic analogs of zoledronate and risedronate inhibit Plasmodium geranylgeranyl diphosphate synthase (GGPPS) and exhibit potent antimalarial activity, Proc. Natl. Acad. Sci. U. S. A. 109, 4058-4063. DOI: https://doi.org/10.1073/pnas.1118215109

[24] Tonhosolo, R., D'Alexandri, F. L., Genta, F. A., Wunderlich, G., Gozzo, F. C., Eberlin, M. N., Peres, V. J., Kimura, E. A., and Katzin, A. M. (2005) Identification, molecular 
1242

1243

1244

1245

1246

1247

1248

1249

1250

1251

1252

1253

1254

1255

1256

1257

1258

1259

1260

1261

1262

1263

cloning and functional characterization of an octaprenyl pyrophosphate synthase in intraerythrocytic stages of Plasmodium falciparum, Biochem. J. 392, 117-126. DOI: https://doi.org/10.1042/BJ20050441

[25] Nair, S. C., Brooks, C. F., Goodman, C. D., Sturm, A., McFadden, G. I., Sundriyal, S., Anglin, J. L., Song, Y., Moreno, S. N., and Striepen, B. (2011) Apicoplast isoprenoid precursor synthesis and the molecular basis of fosmidomycin resistance in Toxoplasma gondii, J. Exp. Med. 208, 1547-1559. DOI: https://doi.org/10.1084/jem.20110039

[26] Bowman, J. D., Merino, E. F., Brooks, C. F., Striepen, B., Carlier, P. R., and Cassera, M. B. (2014) Antiapicoplast and gametocytocidal screening to identify the mechanisms of action of compounds within the malaria box, Antimicrob. Agents Chemother. 58, 811819. DOI: https://doi.org/10.1128/AAC.01500-13

[27] Goodman, C. D., and McFadden, G. I. (2014) Ycf93 (Orf105), a small apicoplast-encoded membrane protein in the relict plastid of the malaria parasite Plasmodium falciparum that is conserved in Apicomplexa, PLoS One 9, e91178. DOI:

https://doi.org/10.1371/journal.pone.0091178

[28] Waller, R. F., Reed, M. B., Cowman, A. F., and McFadden, G. I. (2000) Protein trafficking to the plastid of Plasmodium falciparum is via the secretory pathway, EMBO J. 19, 17941802.

[29] Swift, R. P., Rajaram, K., Liu, H. B., Dziedzic, A., Jedlicka, A. E., Roberts, A. D., Matthews, K. A., Jhun, H., Bumpus, N. N., Tewari, S. G., Wallqvist, A., and Prigge, S. T. (2020) A mevalonate bypass system facilitates elucidation of plastid biology in malaria parasites, PLoS Pathog. 16, e1008316. DOI: https://doi.org/10.1371/journal.ppat.1008316

[30] Howe, R., Kelly, M., Jimah, J., Hodge, D., and Odom, A. R. (2013) Isoprenoid biosynthesis inhibition disrupts Rab5 localization and food vacuolar integrity in Plasmodium falciparum, Eukaryot. Cell 12, 215-223. DOI: https://doi.org/10.1128/EC.00073-12

[31] Zhang, B., Watts, K. M., Hodge, D., Kemp, L. M., Hunstad, D. A., Hicks, L. M., and Odom, A. R. (2011) A second target of the antimalarial and antibacterial agent fosmidomycin revealed by cellular metabolic profiling, Biochemistry 50, 3570-3577. DOI: https://doi.org/10.1021/bi200113y

[32] Cassera, M. B., Gozzo, F. C., D'Alexandri, F. L., Merino, E. F., del Portillo, H. A., Peres, V. J., Almeida, I. C., Eberlin, M. N., Wunderlich, G., Wiesner, J., Jomaa, H., Kimura, E. A., and Katzin, A. M. (2004) The methylerythritol phosphate pathway is functionally active in all intraerythrocytic stages of Plasmodium falciparum, J. Biol. Chem. 279, 5174951759. DOI: https://doi.org/10.1074/jbc.M408360200

[33] Phillips, M. A., Gujjar, R., Malmquist, N. A., White, J., El Mazouni, F., Baldwin, J., and Rathod, P. K. (2008) Triazolopyrimidine-based dihydroorotate dehydrogenase inhibitors with potent and selective activity against the malaria parasite Plasmodium falciparum, $J$. Med. Chem. 51, 3649-3653. DOI: https://doi.org/10.1021/jm8001026

[34] Fry, M., and Pudney, M. (1992) Site of action of the antimalarial hydroxynaphthoquinone, 2-[trans-4-(4'-chlorophenyl) cyclohexyl]-3-hydroxy-1,4-naphthoquinone (566C80), Biochem. Pharmacol. 43, 1545-1553. DOI: https://doi.org/10.1016/0006-2952(92)90213$\underline{3}$

[35] Mamoun, C. B., Gluzman, I. Y., Goyard, S., Beverley, S. M., and Goldberg, D. E. (1999) A set of independent selectable markers for transfection of the human malaria parasite 
1278

1279

1280

1281

1282

1283

1284

1285

1286

1287

1288

1289

1290

1291

1292

1293

1294

1295

1296

1297

1298

1299

1300

1301

1302

1303

1304

1305

1306

1307

1308

Plasmodium falciparum, Proc. Natl. Acad. Sci. U. S. A. 96, 8716-8720. DOI: https://doi.org/10.1073/pnas.96.15.8716

[36] Fidock, D. A., and Wellems, T. E. (1997) Transformation with human dihydrofolate reductase renders malaria parasites insensitive to WR99210 but does not affect the intrinsic activity of proguanil, Proc. Natl. Acad. Sci. U. S. A. 94, 10931-10936. DOI: https://doi.org/10.1073/pnas.94.20.10931

[37] Swift, R. P., Rajaram, K., Keutcha, C., Liu, H. B., Kwan, B., Dziedzic, A., Jedlicka, A. E., and Prigge, S. T. (2020) The NTP generating activity of pyruvate kinase II is critical for apicoplast maintenance in Plasmodium falciparum, Elife 9. DOI: https://doi.org/10.7554/eLife.50807

[38] Dahl, E. L., Shock, J. L., Shenai, B. R., Gut, J., DeRisi, J. L., and Rosenthal, P. J. (2006) Tetracyclines specifically target the apicoplast of the malaria parasite Plasmodium falciparum, Antimicrob. Agents Chemother. 50, 3124-3131. DOI: https://doi.org/10.1128/AAC.00394-06

[39] van Dooren, G. G., Marti, M., Tonkin, C. J., Stimmler, L. M., Cowman, A. F., and McFadden, G. I. (2005) Development of the endoplasmic reticulum, mitochondrion and apicoplast during the asexual life cycle of Plasmodium falciparum, Mol. Microbiol. 57, 405-419. DOI: https://doi.org/10.1111/j.1365-2958.2005.04699.x

[40] Persson, B. C., Esberg, B., Olafsson, O., and Bjork, G. R. (1994) Synthesis and function of isopentenyl adenosine derivatives in tRNA, Biochimie 76, 1152-1160. DOI: https://doi.org/10.1016/0300-9084(94)90044-2

[41] Esberg, B., Leung, H. C., Tsui, H. C., Bjork, G. R., and Winkler, M. E. (1999) Identification of the miaB gene, involved in methylthiolation of isopentenylated A37 derivatives in the tRNA of Salmonella typhimurium and Escherichia coli, J. Bacteriol. 181, 7256-7265. DOI: https://doi.org/10.1128/JB.181.23.7256-7265.1999

[42] Boucher, M. J., Ghosh, S., Zhang, L., Lal, A., Jang, S. W., Ju, A., Zhang, S., Wang, X., Ralph, S. A., Zou, J., Elias, J. E., and Yeh, E. (2018) Integrative proteomics and bioinformatic prediction enable a high-confidence apicoplast proteome in malaria parasites, PLoS Biol. 16, e2005895. DOI: https://doi.org/10.1371/journal.pbio.2005895

[43] Bushell, E., Gomes, A. R., Sanderson, T., Anar, B., Girling, G., Herd, C., Metcalf, T., Modrzynska, K., Schwach, F., Martin, R. E., Mather, M. W., McFadden, G. I., Parts, L., Rutledge, G. G., Vaidya, A. B., Wengelnik, K., Rayner, J. C., and Billker, O. (2017) Functional Profiling of a Plasmodium Genome Reveals an Abundance of Essential Genes, Cell 170, 260-272 e268. DOI: https://doi.org/10.1016/j.cell.2017.06.030

[44] Zhang, M., Wang, C., Otto, T. D., Oberstaller, J., Liao, X., Adapa, S. R., Udenze, K., Bronner, I. F., Casandra, D., Mayho, M., Brown, J., Li, S., Swanson, J., Rayner, J. C., Jiang, R. H. Y., and Adams, J. H. (2018) Uncovering the essential genes of the human malaria parasite Plasmodium falciparum by saturation mutagenesis, Science 360. DOI: https://doi.org/10.1126/science.aap7847

[45] Kellogg, B. A., and Poulter, C. D. (1997) Chain elongation in the isoprenoid biosynthetic pathway, Curr. Opin. Chem. Biol. 1, 570-578. DOI: https://doi.org/10.1016/s13675931(97)80054-3

[46] Poulter, C. D. (2006) Farnesyl diphosphate synthase. A paradigm for understanding structure and functional relationships in E-polyprenyl diphosphate synthases, Phytochem. Rev. 5, 17-26. DOI: https://doi.org/10.1007/s11101-005-4887-1 
[47] Thulasiram, H. V., and Poulter, C. D. (2006) Farnesyl diphosphate synthase: The art of compromise between substrate selectivity and stereoselectivity, J. Am. Chem. Soc. 128, 15819-15823. DOI: https://doi.org/10.1021/ja065573b

[48] Tarshis, L. C., Proteau, P. J., Kellogg, B. A., Sacchettini, J. C., and Poulter, C. D. (1996) Regulation of product chain length by isoprenyl diphosphate synthases, Proc. Natl. Acad. Sci. U. S. A. 93, 15018-15023. DOI: https://doi.org/10.1073/pnas.93.26.15018

[49] Jordao, F. M., Gabriel, H. B., Alves, J. M., Angeli, C. B., Bifano, T. D., Breda, A., de Azevedo, M. F., Basso, L. A., Wunderlich, G., Kimura, E. A., and Katzin, A. M. (2013) Cloning and characterization of bifunctional enzyme farnesyl diphosphate/geranylgeranyl diphosphate synthase from Plasmodium falciparum, Malar. J. 12, 184. DOI: https://doi.org/10.1186/1475-2875-12-184

[50] Artz, J. D., Wernimont, A. K., Dunford, J. E., Schapira, M., Dong, A., Zhao, Y., Lew, J., Russell, R. G., Ebetino, F. H., Oppermann, U., and Hui, R. (2011) Molecular characterization of a novel geranylgeranyl pyrophosphate synthase from Plasmodium parasites, J. Biol. Chem. 286, 3315-3322. DOI: https://doi.org/10.1074/jbc.M109.027235 [51] Boratyn, G. M., Camacho, C., Cooper, P. S., Coulouris, G., Fong, A., Ma, N., Madden, T. L., Matten, W. T., McGinnis, S. D., Merezhuk, Y., Raytselis, Y., Sayers, E. W., Tao, T., Ye, J., and Zaretskaya, I. (2013) BLAST: a more efficient report with usability improvements, Nucleic Acids Res. 41, W29-33. DOI: https://doi.org/10.1093/nar/gkt282

[52] Zimmermann, L., Stephens, A., Nam, S. Z., Rau, D., Kubler, J., Lozajic, M., Gabler, F., Soding, J., Lupas, A. N., and Alva, V. (2018) A Completely Reimplemented MPI Bioinformatics Toolkit with a New HHpred Server at its Core, J. Mol. Biol. 430, $2237-$ 2243. DOI: https://doi.org/10.1016/j.jmb.2017.12.007

[53] Tonhosolo, R., D'Alexandri, F. L., de Rosso, V. V., Gazarini, M. L., Matsumura, M. Y., Peres, V. J., Merino, E. F., Carlton, J. M., Wunderlich, G., Mercadante, A. Z., Kimura, E. A., and Katzin, A. M. (2009) Carotenoid biosynthesis in intraerythrocytic stages of Plasmodium falciparum, J. Biol. Chem. 284, 9974-9985. DOI: https://doi.org/10.1074/jbc.M807464200

[54] Foth, B. J., Ralph, S. A., Tonkin, C. J., Struck, N. S., Fraunholz, M., Roos, D. S., Cowman, A. F., and McFadden, G. I. (2003) Dissecting apicoplast targeting in the malaria parasite Plasmodium falciparum, Science 299, 705-708.

[55] Sigala, P. A., Crowley, J. R., Henderson, J. P., and Goldberg, D. E. (2015) Deconvoluting heme biosynthesis to target blood-stage malaria parasites, Elife 4. DOI: https://doi.org/10.7554/eLife.09143

[56] Ghorbal, M., Gorman, M., Macpherson, C. R., Martins, R. M., Scherf, A., and Lopez-Rubio, J. J. (2014) Genome editing in the human malaria parasite Plasmodium falciparum using the CRISPR-Cas9 system, Nat. Biotechnol. 32, 819-821. DOI: https://doi.org/10.1038/nbt.2925

[57] Ganesan, S. M., Falla, A., Goldfless, S. J., Nasamu, A. S., and Niles, J. C. (2016) Synthetic RNA-protein modules integrated with native translation mechanisms to control gene expression in malaria parasites, Nat. Commun. 7, 10727. DOI: https://doi.org/10.1038/ncomms10727

[58] Maruthi, M., Ling, L., Zhou, J., and Ke, H. (2020) Dispensable Role of Mitochondrial Fission Protein 1 (Fis1) in the Erythrocytic Development of Plasmodium falciparum, mSphere 5. DOI: https://doi.org/10.1128/mSphere.00579-20 
1354

1355

1356

1357

1358

1359

1360

1361

1362

1363

1364

1365

1366

1367

1368

1369

1370

1371

1372

1373

1374

1375

1376

1377

1378

1379

1380

1381

1382

1383

1384

1385

1386

1387

1388

1389

1390

1391

1392

1393

1394

1395

1396

1397

[59] Gabriel, H. B., Silva, M. F., Kimura, E. A., Wunderlich, G., Katzin, A. M., and Azevedo, M. F. (2015) Squalestatin is an inhibitor of carotenoid biosynthesis in Plasmodium falciparum, Antimicrob. Agents Chemother. 59, 3180-3188. DOI: https://doi.org/10.1128/AAC.04500-14

[60] Bouvier, F., Rahier, A., and Camara, B. (2005) Biogenesis, molecular regulation and function of plant isoprenoids, Prog. Lipid Res. 44, 357-429. DOI:

https://doi.org/10.1016/j.plipres.2005.09.003

[61] Zhou, X., Welsch, R., Yang, Y., Alvarez, D., Riediger, M., Yuan, H., Fish, T., Liu, J., Thannhauser, T. W., and Li, L. (2015) Arabidopsis OR proteins are the major posttranscriptional regulators of phytoene synthase in controlling carotenoid biosynthesis, Proc. Natl. Acad. Sci. U. S. A. 112, 3558-3563. DOI:

https://doi.org/10.1073/pnas.1420831112

[62] Iwata-Reuyl, D., Math, S. K., Desai, S. B., and Poulter, C. D. (2003) Bacterial phytoene synthase: molecular cloning, expression, and characterization of Erwinia herbicola phytoene synthase, Biochemistry 42, 3359-3365. DOI: https://doi.org/10.1021/bi0206614

[63] Nagashima, S., Kamimura, A., Shimizu, T., Nakamura-Isaki, S., Aono, E., Sakamoto, K., Ichikawa, N., Nakazawa, H., Sekine, M., Yamazaki, S., Fujita, N., Shimada, K., Hanada, S., and Nagashima, K. V. (2012) Complete genome sequence of phototrophic betaproteobacterium Rubrivivax gelatinosus IL144, J. Bacteriol. 194, 3541-3542. DOI: https://doi.org/10.1128/JB.00511-12

[64] Cunningham, F. X., Jr., and Gantt, E. (2005) A study in scarlet: enzymes of ketocarotenoid biosynthesis in the flowers of Adonis aestivalis, Plant J. 41, 478-492. DOI: https://doi.org/10.1111/j.1365-313X.2004.02309.x

[65] McFadden, G. I., and Yeh, E. (2016) The apicoplast: now you see it, now you don't, Int. J. Parasitol. DOI: https://doi.org/10.1016/j.ijpara.2016.08.005

[66] Akhtar, T. A., Surowiecki, P., Siekierska, H., Kania, M., Van Gelder, K., Rea, K. A., Virta, L. K. A., Vatta, M., Gawarecka, K., Wojcik, J., Danikiewicz, W., Buszewicz, D., Swiezewska, E., and Surmacz, L. (2017) Polyprenols Are Synthesized by a Plastidial cisPrenyltransferase and Influence Photosynthetic Performance, Plant Cell 29, 1709-1725. DOI: https://doi.org/10.1105/tpc.16.00796

[67] Joyard, J., Ferro, M., Masselon, C., Seigneurin-Berny, D., Salvi, D., Garin, J., and Rolland, N. (2009) Chloroplast proteomics and the compartmentation of plastidial isoprenoid biosynthetic pathways, Mol. Plant 2, 1154-1180. DOI: https://doi.org/10.1093/mp/ssp088

[68] Van Gelder, K., Rea, K. A., Virta, L. K. A., Whitnell, K. L., Osborn, M., Vatta, M., Khozin, A., Skorupinska-Tudek, K., Surmacz, L., and Akhtar, T. A. (2018) Medium-Chain Polyprenols Influence Chloroplast Membrane Dynamics in Solanum lycopersicum, Plant Cell Physiol. 59, 2350-2365. DOI: https://doi.org/10.1093/pcp/pcy157

[69] Kelly, M., Su, C. Y., Schaber, C., Crowley, J. R., Hsu, F. F., Carlson, J. R., and Odom, A. R. (2015) Malaria parasites produce volatile mosquito attractants, mBio 6. DOI: https://doi.org/10.1128/mBio.00235-15

[70] Emami, S. N., Lindberg, B. G., Hua, S., Hill, S. R., Mozuraitis, R., Lehmann, P., Birgersson, G., Borg-Karlson, A. K., Ignell, R., and Faye, I. (2017) A key malaria metabolite modulates vector blood seeking, feeding, and susceptibility to infection, Science 355, 1076-1080. DOI: https://doi.org/10.1126/science.aah4563 
1398

1399

1400

1401

1402

1403

1404

1405

1406

1407

1408

1409

1410

1411

1412

1413

1414

1415

1416

1417

1418

1419

1420

1421

1422

1423

1424

1425

1426

1427

1428

1429

1430

1431

1432

1433

1434

1435

1436

1437

1438

1439

1440

1441

1442

1443

[71] Miller, J. J., and Odom John, A. R. (2020) The Malaria Metabolite HMBPP Does Not Trigger Erythrocyte Terpene Release, ACS Infect. Dis. 6, 2567-2572. DOI: https://doi.org/10.1021/acsinfecdis.0c00548

[72] Couto, A. S., Kimura, E. A., Peres, V. J., Uhrig, M. L., and Katzin, A. M. (1999) Active isoprenoid pathway in the intra-erythrocytic stages of Plasmodium falciparum: presence of dolichols of 11 and 12 isoprene units, Biochem. J. 341 ( Pt 3), 629-637.

[73] Zimbres, F. M., Valenciano, A. L., Merino, E. F., Florentin, A., Holderman, N. R., He, G., Gawarecka, K., Skorupinska-Tudek, K., Fernandez-Murga, M. L., Swiezewska, E., Wang, X., Muralidharan, V., and Cassera, M. B. (2020) Metabolomics profiling reveals new aspects of dolichol biosynthesis in Plasmodium falciparum, Sci. Rep. 10, 13264. DOI: https://doi.org/10.1038/s41598-020-70246-0

[74] Hartley, M. D., and Imperiali, B. (2012) At the membrane frontier: a prospectus on the remarkable evolutionary conservation of polyprenols and polyprenyl-phosphates, Arch. Biochem. Biophys. 517, 83-97. DOI: https://doi.org/10.1016/j.abb.2011.10.018

[75] Swiezewska, E., and Danikiewicz, W. (2005) Polyisoprenoids: structure, biosynthesis and function, Prog. Lipid Res. 44, 235-258. DOI:

https://doi.org/10.1016/j.plipres.2005.05.002

[76] Botte, C. Y., Yamaryo-Botte, Y., Rupasinghe, T. W., Mullin, K. A., MacRae, J. I., Spurck, T. P., Kalanon, M., Shears, M. J., Coppel, R. L., Crellin, P. K., Marechal, E., McConville, M. J., and McFadden, G. I. (2013) Atypical lipid composition in the purified relict plastid (apicoplast) of malaria parasites, Proc. Natl. Acad. Sci. U. S. A. 110, 75067511. DOI: https://doi.org/10.1073/pnas.1301251110

[77] Tonkin, C. J., van Dooren, G. G., Spurck, T. P., Struck, N. S., Good, R. T., Handman, E., Cowman, A. F., and McFadden, G. I. (2004) Localization of organellar proteins in Plasmodium falciparum using a novel set of transfection vectors and a new immunofluorescence fixation method, Mol. Biochem. Parasitol. 137, 13-21.

[78] Gallagher, J. R., and Prigge, S. T. (2010) Plasmodium falciparum acyl carrier protein crystal structures in disulfide-linked and reduced states and their prevalence during blood stage growth, Proteins 78, 575-588. DOI: https://doi.org/10.1002/prot.22582

[79] Ganesan, S. M., Morrisey, J. M., Ke, H., Painter, H. J., Laroiya, K., Phillips, M. A., Rathod, P. K., Mather, M. W., and Vaidya, A. B. (2011) Yeast dihydroorotate dehydrogenase as a new selectable marker for Plasmodium falciparum transfection, Mol. Biochem. Parasitol. 177, 29-34.

[80] Klemba, M., Beatty, W., Gluzman, I., and Goldberg, D. E. (2004) Trafficking of plasmepsin II to the food vacuole of the malaria parasite Plasmodium falciparum, J. Cell Biol. 164, 47-56.

[81] Beck, J. R., Muralidharan, V., Oksman, A., and Goldberg, D. E. (2014) PTEX component HSP101 mediates export of diverse malaria effectors into host erythrocytes, Nature 511, 592-595. DOI: https://doi.org/10.1038/nature13574

[82] Balu, B., Shoue, D. A., Fraser, M. J., and Adams, J. H. (2005) High-efficiency transformation of Plasmodium falciparum by the lepidopteran transposable element piggyBac, Proc. Natl. Acad. Sci. U. S. A. 102, 16391-16396. DOI: https://doi.org/Doi 10.1073/Pnas.0504679102

[83] Spillman, N. J., Beck, J. R., Ganesan, S. M., Niles, J. C., and Goldberg, D. E. (2017) The chaperonin TRiC forms an oligomeric complex in the malaria parasite cytosol, Cell Microbiol. 19. DOI: https://doi.org/10.1111/cmi.12719 
1444 [84] Schmittgen, T. D., and Livak, K. J. (2008) Analyzing real-time PCR data by the comparative C(T) method, Nat. Protoc. 3, 1101-1108. DOI: https://doi.org/10.1038/nprot.2008.73

[85] Rajaram, K., Liu, H. B., and Prigge, S. T. (2020) Redesigned TetR-Aptamer System To Control Gene Expression in Plasmodium falciparum, mSphere 5. DOI:

1453 https://doi.org/10.1128/mSphere.00457-20

[86] Rivera, S. M., Christou, P., and Canela-Garayoa, R. (2014) Identification of carotenoids using mass spectrometry, Mass Spectrom. Rev. 33, 353-372. DOI: https://doi.org/10.1002/mas. 21390 